\title{
A Discrete Huber-braun Neuron Model: From Nodal Properties to Network Performance
}

\section{Shaoba He}

Central South University

Karthikeyan Rajagopal

Chennai Institute of Technology

Anitha Karthikeyan ( $\nabla$ anitha.karthikeyan@tdtu.edu.vn )

Ton Duc Thang University https://orcid.org/0000-0001-6485-4687

\section{Ashokkumar Srinivasan}

Chennai Institute of Technology

\section{Research Article}

Keywords: discrete neuron, chaos, bifurcation, spiral waves, noise effects

Posted Date: March 11th, 2021

DOl: https://doi.org/10.21203/rs.3.rs-243100/v1

License: (9) This work is licensed under a Creative Commons Attribution 4.0 International License. Read Full License 


\title{
A discrete Huber-Braun neuron Model: From nodal properties to network performance
}

\author{
Shaobo He ${ }^{a}$, Karthikeyan Rajagopal ${ }^{b}$, Anitha Karthikeyan ${ }^{c^{*}}$, Ashokkumar Srinivasan ${ }^{\mathrm{d}}$ \\ ${ }^{a}$ School of Physics and Electronics, Central South University, Changsha, 410083, China \\ heshaobo@csu.edu.cn \\ ${ }^{b, d}$ Center for Nonlinear Systems, Chennai Institute of Technology, India \\ ${ }^{b}$ karthikeyan.rajagopal@citchennai.net \\ dashokkumar.srinivasan@citchennai.net \\ ${ }^{c}$ Nonlinear Systems and Applications, Faculty of Electrical and Electronics Engineering, Ton \\ Duc Thang University, Ho Chi Minh City, Vietnam. \\ anitha.karthikeyan@tdtu.edu.vn \\ *Corresponding author
}

\begin{abstract}
Many of the well-known neuron models are continuous time systems with complex mathematical definitions. Literatures have shown that a discrete mathematical model can effectively replicate the complete dynamical behaviour of a neuron with much reduced complexity. Hence, we propose a new discrete neuron model derived from the Huber-Braun neuron with two additional slow and subthreshold currents alongside the ion channel currents. We have also introduced temperature dependent ion channels to study its effects on the firing pattern of the neuron. With bifurcation and Lyapunov exponents we showed the chaotic and periodic regions of the discrete model. Further to study the complexity of the neuron model, we have used the sample entropy algorithm. Though the individual neuron analysis gives us an idea about the dynamical properties, it's the collective behaviour which decides the overall behavioural pattern of the neuron. Hence, we investigate the spatiotemporal behaviour of the discrete neuron model in single- and two-layer network. We have considered noise and obstacles as the two important factor which changes the excitability of the neurons in the network. When there is no noise or obstacle, the network display simple wave propagation with highly excitable neurons. Literatures have shown that spiral waves can play a positive role in breaking through quiescent areas of the brain as a pacemaker by creating a coherence resonance behaviour. Hence, we are interested in studying the induced spiral waves in the network. In this condition when an obstacle is introduced the wave propagation is disturbed and we could see multiple wave re-entry and spiral waves. Now when we consider only noise with no obstacle, for selected noise variances the network supports wave re-entry. By introducing an obstacle in this noisy network, the re-entry soon disappears, and the network soon enters idle state with no resetting. In a two-layer network when the obstacle is considered only in one layer and stimulus applied to the layer having the obstacle, the wave re-entry is seen in both the layer though the other layer is not exposed to obstacle. But when both the layers are inserted with an obstacle and stimuli also applied to the layers, they behave like independent layers with no coupling effect. This in a two-layer network stimulus play an important role in spatiotemporal dynamics of the network. Similar noise effects like the single layer network are also seen in the two-layer network.
\end{abstract}


Keywords: discrete neuron; chaos; bifurcation; spiral waves; noise effects.

\section{Introduction}

Investigating the local dynamics of neurons is vital in understanding their oscillatory behaviour but will not be useful in analysing their collective dynamics. To mention, phenomena such as synchronization, chimera states, Spiral waves etc., cannot be studied with such low population neuron models. Hence in the recent years the research throng has shifted towards the neuronal network modelling for biological realism. The challenge starts from modelling the action potentials such as frequency, inter spike intervals (ISI), bursting or frequency of bursting. A map-based neuron is identified with effective in computationally feasible and tailored for investigating phenomenological behaviours [1-5]. The dynamical map can be defined as dynamical systems with discrete space and discrete time, but with continuous state variables. In [4] an approach is presented with simplification of continuous time differential equation models into discrete time and keeping the desired properties. The early work on Map-based models enumerated with advantages such as independency from integration and integration step adjustments. But the existence of piecewise linear function affects the performance particularly phase plane analysis[3, $6,7]$. Formulation of network topology for combining the discrete time elements with synapse model is the other challenge. Among various types, diffusive coupling models $[2,8]$ found its attraction because it uses synaptic current through a time dependent (or not) conductance. In [2, 9] Rulkov and De vries investigated the synchronization and existence of spatiotemporal chaos with neuron coupling strength. The influence of noise on such maps are studied intensively and confirmed that the spatiotemporal pattern generation and propagation are affected with noise [10] and it can be suppressed with different techniques [11-14]. [1, 2, 7, 15, 16] extended this model to study the chaotic phase synchronization and burst synchronization and proved that discrete maps are superior to study the network behaviour of neuronal system. The major challenge is, system stability not matching with the map stability and results with restriction to study the delay nature and temperature effects on the neuron synapse and network [17-22].

In $[12,23]$ Huber Braun derived Temperature dependent Hodgkin-Huxley-type neurons and presented various dynamic nature of the neuronal system under different temperature conditions, which other models fails to show. The model ensembled with two slow, subthreshold currents along with the leak and channel currents and henceforth called Huber Braun (HB) neurons. The model can expose different spiking patterns and bursting and chaotic nature [1, 24, 25]. The significant impression of noise on neuronal network is extensively studied and pronounced white noise of Box-Muller type is effective for exploring wave generation and propagation phenomena [10-12, 14, 15, 26-28]. Similarly, the experimental evidences show that the transmission of signals between neurons in single layer is considerably different from neurons in different layers [29-32], it extends the scope of study from single layer into multilayer network.

The advent of irregular patterns in electrically coupled networks are studied in horde of literatures $[6,7,10,11,15,16,28,33,34]$ but many not considered the presence of obstacles and very few discussed influences of the obstacle size and orientation [35-43]. Even those are not considered multilayer networking strategy. 
A rotating spiral wave with its frequency higher than the frequency of a pacemaker often disturbs the regularity of heartbeat and thus causing arrhythmias and cardiac death. More than a spiral seed the danger imposed by multiple broken spiral waves are more because of its multiple frequency of rotation which increases the turbulent activity of the heart tissues and thus causing atrial fibrillation [44]. This unsynchronised mechanical contrition of the heart muscles causes the irregular pumping of the blood. Such situations can often arise because of the heterogeneity and obstacles [45] but this theory was not completely proven in the literatures as the relation between cardiac arrhythmias and reduced excitability is still a topic of debate. Spiral wave studies were conducted on ODE neuron models and literatures have successfully shows the effects of such spiral waves and the interlink between the emergence of spiral waves and tissue excitability. But such analysis needs complex computations and longer simulation times as resetting process required by the neurons are induced depending on the step size of the solver used. But when using a discrete neuron model such complexities are resolved as the resetting happens in every step without loss of the actual behaviour of the neuron.

Motivated by this in the present study we propose a discrete HB neuron model and investigating the behavioural patterns of the discrete neuron such as periodic and chaotic bursting. Further to study the network behaviour we used a 2D lattice of the discrete HD neurons and we considered the white noise influence and studied the network kinetics with single and two layers for different stimulus conditions. The paper organized as (i) Formulation of Discrete HB neuron model (ii) Dynamic analysis and bifurcation plots for parameter variations (iii) Complexity analysis based on the sample entropy (SampEn) algorithm (iv) Spiral wave generation and propagation without obstacle (v) Effect of obstacle for different size and orientation (vi) Effects of obstacle in spatiotemporal behaviour with different size and orientation (vii) Effects of noise in spatiotemporal behaviour with single and two layers supplied with periodic stimulus

\section{Discrete Huber-Braun neuron (DHB) model:}

The continuous time model of a Huber-Braun type neuron which is the modification of the wellknown Hodgkin-Huxley models derived by including two slow, subthreshold currents along with the leak and channel currents was discussed in [27]. But many literatures have shown that discrete neuron maps are valid phenomenological neuron models [19]. Normally discretisation can be applied with respect to space, time and field in neuron models. We are interested in applying discretisation in time and propose a new discrete neuron model derived from the continuous time Huber-Braun type neurons. The new neuron map can be called as Discrete Huber-Braun neuron (DHB) model whose mathematical model is defined by 


$$
\begin{aligned}
& V(k+1)=\frac{-I_{l}(k)-I_{d}(k)-I_{r}(k)-I_{s d}(k)-I_{s r}(k)}{C_{M}} \\
& a_{r}(k+1)=\phi(T) \frac{\left(a_{r \infty}(k)-a_{r}(\mathrm{k})\right)}{\tau_{\mu}} \\
& a_{s d}(k+1)=\phi(T) \frac{\left(a_{s d \infty}(k)-a_{s d}(\mathrm{k})\right)}{\tau_{\mu}} \\
& a_{s r}(k+1)=\phi(T) \frac{\left(-\eta \mathrm{I}_{s d}(k)-k a_{s r}(\mathrm{k})\right)}{\tau_{s r}}
\end{aligned}
$$

where the currents are defined by the relations with $I_{l}$ defining the leak current, $I_{d}$ and $I_{r}$ representing depolarising and repolarising currents, $I_{s d}$ and $I_{s r}$ representing the sub-threshold depolarising and repolarising currents respectively.

$$
\begin{aligned}
& I_{l}(k)=g_{l}\left(V(k)-V_{l}\right) \\
& I_{d}(k)=\rho(T) g_{d} a_{d}(k)\left(V(k)-V_{d}\right) \\
& I_{r}(k)=\rho(T) g_{r} a_{r}(k)\left(V(k)-V_{r}\right) \\
& I_{s d}(k)=\rho(T) g_{s d} a_{s d}(k)\left(V(k)-V_{s d}\right) \\
& I_{s r}(k)=\rho(T) g_{s r} r_{s r}(k)\left(V(k)-V_{s r}\right)
\end{aligned}
$$

In all the current and function definitions the suffix $d$ denotes depolarising and $r$ denotes repolarising. The slow depolarising and repolarising parameters and functions are defined with a suffix $s d$ and $s r$ respectively. The steady state activation functions are defined by the relations,

\begin{tabular}{|l|}
$\mathrm{a}_{r o \infty}(k)=\frac{1}{1+\exp \left(-s_{r}\left(V(k)-V_{0 r}\right)\right.}$ \\
$\mathrm{a}_{s d \infty}(k)=\frac{1}{1+\exp \left(-s_{s d}\left(V(k)-V_{0 s d}\right)\right.}$ \\
$\mathrm{a}_{d}(k)=\frac{1}{1+\exp \left(-s_{d}\left(V(k)-V_{0 d}\right)\right.}$
\end{tabular}

In order to define the temperature dependencies for the neuron model (1), we use the $Q_{10}$ laws [27] defined by the mathematical relations

$$
\begin{aligned}
& \phi(T)=3^{\left(T-T_{0}\right) / 10^{\circ} \mathrm{C}} \\
& \rho(T)=1.3^{\left(T-T_{0}\right) / 10^{\circ} \mathrm{C}}
\end{aligned}
$$

The system parameters are adopted from [27] and defined as 


\begin{tabular}{|l|}
\hline$V_{s d}=V_{d}=50 m V ; V_{s r}=V_{r}=-90 m V ; V_{l}=-60 m V ; g_{l}=0.1 ; g_{d}=1.5 ; g_{r}=2.0 ;$ \\
$g_{s d}=0.25 ; g_{s r}=0.4\left(\right.$ in $\left.\frac{m s}{c^{2}}\right) ; C_{M}=1\left(\frac{\mu F}{c^{2}}\right) ; \tau_{r}=2 m s ; \tau_{s d}=10 m s ; \tau_{s r}=20 m s ;$ \\
$s_{d}=s_{r}=0.25 ; s_{s d}=0.09 ;$ \\
$V_{0 d}=V_{0 r}=-25 m V ; V_{0 s d}=-40 m V ; \eta=0.012 ; k=0.17 ; D=0.5 ; \mathrm{T}_{0}=25^{\circ} C ;$
\end{tabular}

Set the initial conditions $\left[V(0), a_{r}(0), a_{s d}(0), a_{s r}(0)\right]=[-55,0.01,0,0]$, and $\mathrm{T}=15$ and 11 , the time series of the neural model is shown in Fig.1 (a) and (b), respectively. It shows that the system is periodic when $\mathrm{T}=15$, while is chaotic when $\mathrm{T}=11$.
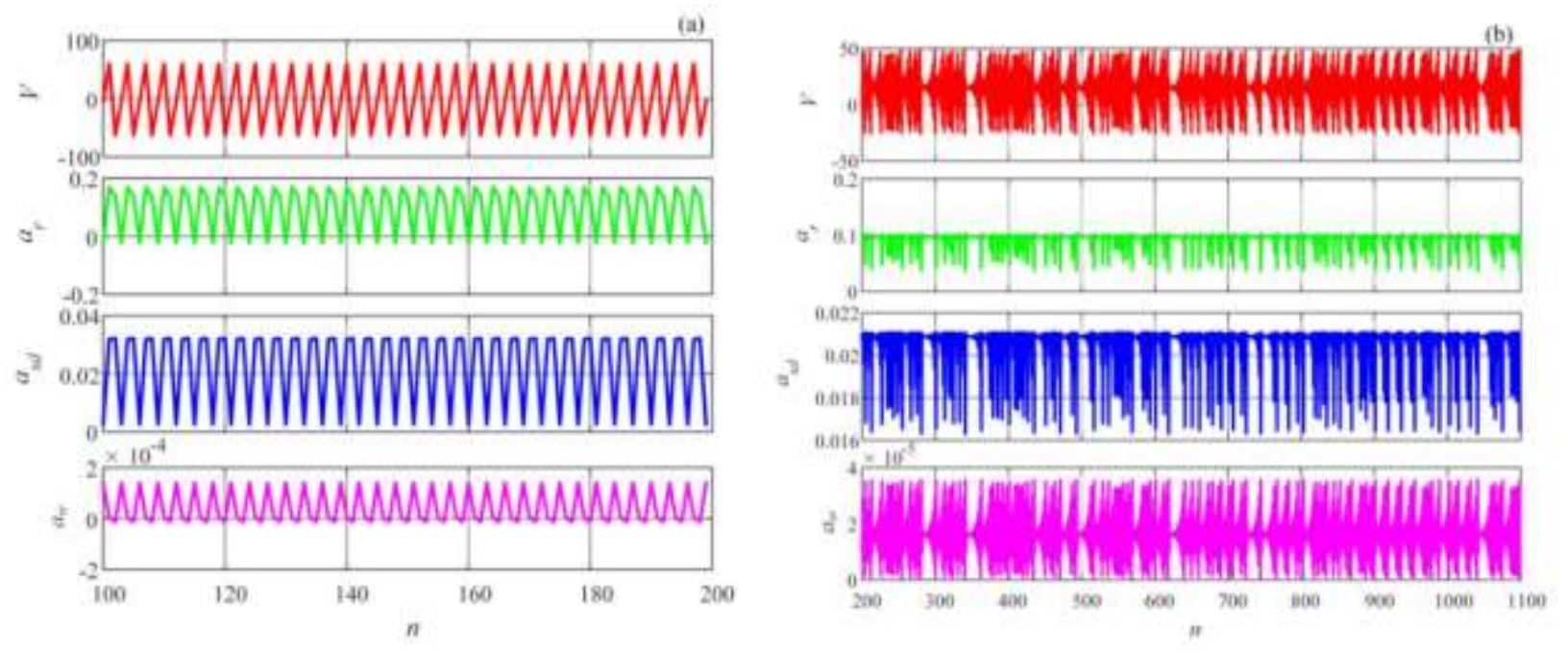

Fig.1: Time series of the system with different parameters. (a) $\mathrm{T}=15$; (b) $\mathrm{T}=11$.

\section{Dynamical properties}

In this section, we focus on the complex dynamics of the system with system parameters gl, gd and $\mathrm{T}$, where bifurcation diagram, Lyapunov exponents (LEs) and sample entropy algorithm are employed. It should be noted that the Jacobian materix of the neural model is obtained used the matlab function "J=jacobian(.)", and the QR decomposition method is applied to estimate Lyapunov exponents. In the figures, since LE3 and LE4 are both smaller than zero, we just illustrate the Lyapunov exponents LE1 and LE2 for simplification. The initial conditions are given as $\left[V(0), a_{r}(0), a_{s d}(0), a_{s r}(0)\right]=[-55,0.01,0,0]$.

\subsection{Bifurcation and Lyapunov exponents}

Firstly, three cases are considered.

Case 1: Let $g_{d}=1.5$ and $\mathrm{T}=11$, vary the parameter gl from 0 to 1.5 with step size of 0.003 . The bifurcation diagram and its corresponding Lyapunovs exponents are shown in Fig.2. It shows that when the value of $g_{l}$ is smaller than 0.2375 , the system is chaotic, then the system goes to chaotic state through a period doubling bifurcation. When $g_{l}>0.72$, the system become to chaotic. 
However, the system becomes stable when $g_{l}$ is close to 1.5 , since all the Lyapunvo exponents are smaller than zero. Obviously, there are also many periodic windows with the increase of $g_{l}$.
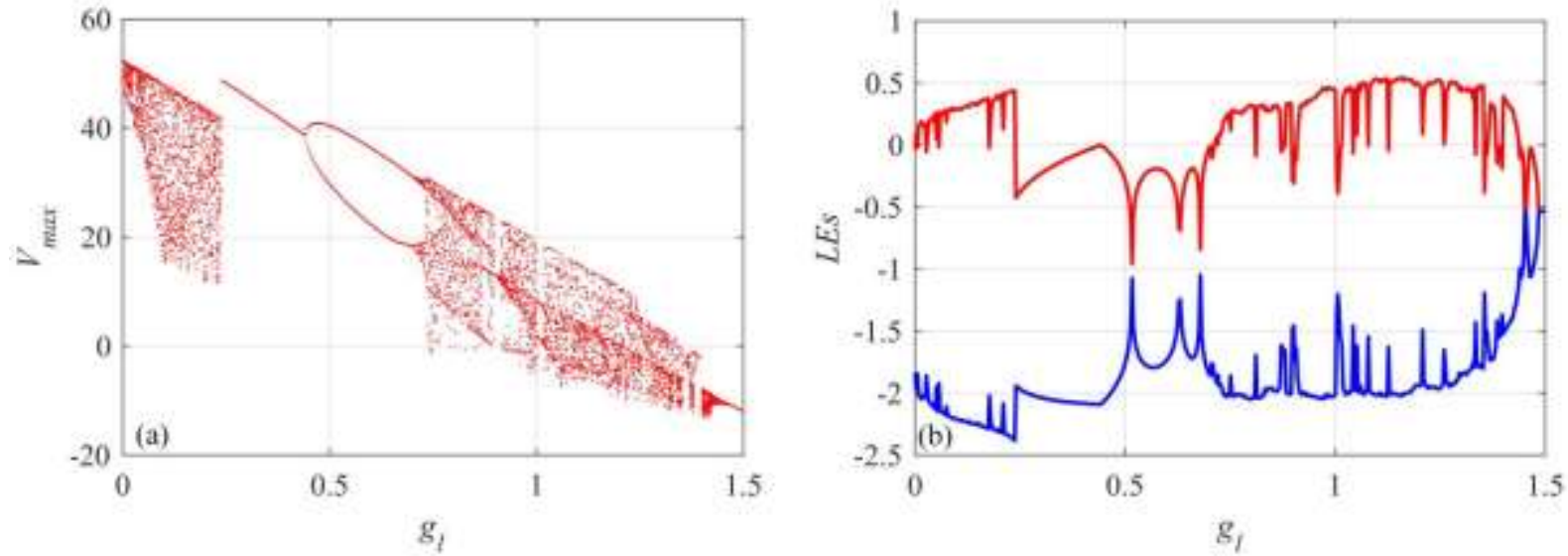

Fig.2: Dynamics of the system with the variation of parameter $g_{l}$. (a) Bifurcation diagram; (b) Lyapunov exponents.

Case 2: Let $g_{l}=0.1$ and $\mathrm{T}=11$, vary the parameter $g_{d}$ from 1 to 2 with step size of 0.002 . The analysis results are illustrated in Fig.3. It shows that the system is chaotic when $g_{d}$ takes values between 1.4 and 1.63, and the system goes to chaos with a period doubling bifurcation route. When $g_{d}>1.63$, the system becomes to stable according to Fig.3-2(b), because the largest Lyapunov exponent is smaller than zero.
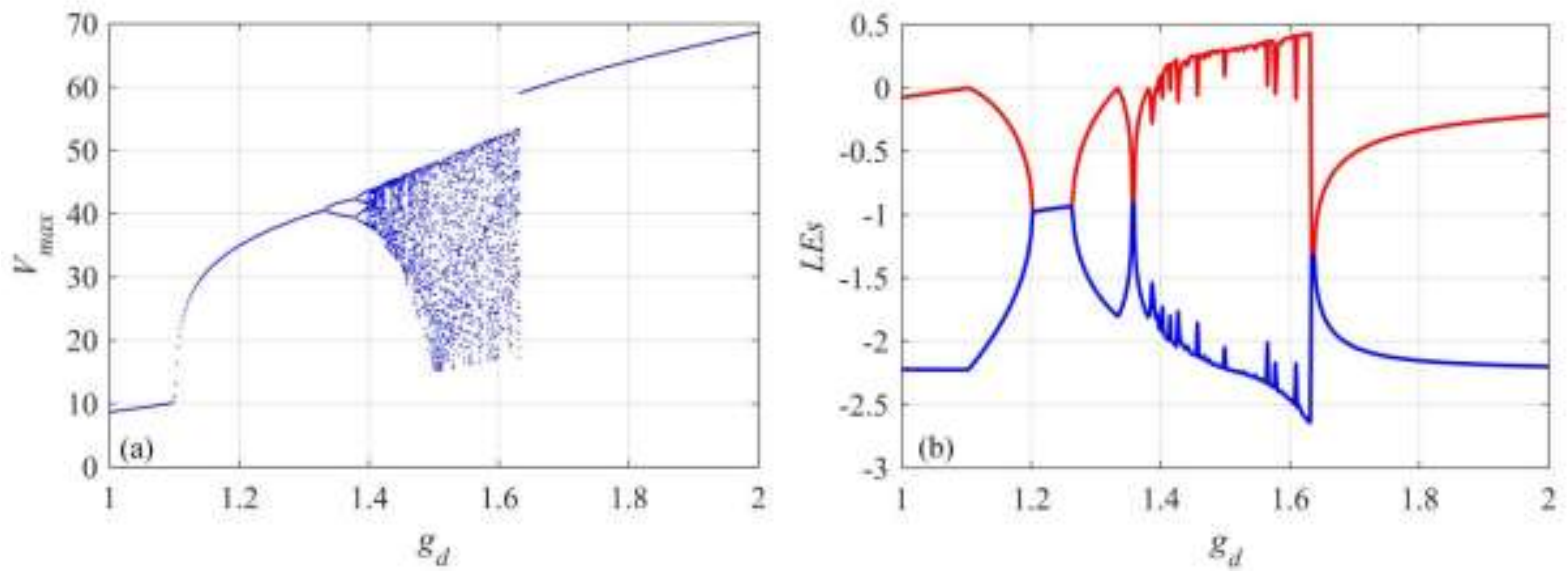

Fig. 3: Dynamics of the system with the variation of parameter gd. (a) Bifurcation diagram; (b) Lyapunov exponents.

Case 3: Let $g_{l}=0.1$ and $g_{d}=1.5$, vary the parameter T from 8 to 28 with step size of 0.0401 . The obtained bifurcation diagram and Lyapunov exponents are shown in Fig.4. It shows that there is a large periodic window when $T \in[12.14,21.1]$. In the left and right side of this periodic window, the system is chaotic. However, the system is non-chaotic when $\mathrm{T}<8.9$. It also shows that there are many small periodic windows with the variations of $\mathrm{T}$ in the chaotic regions. 

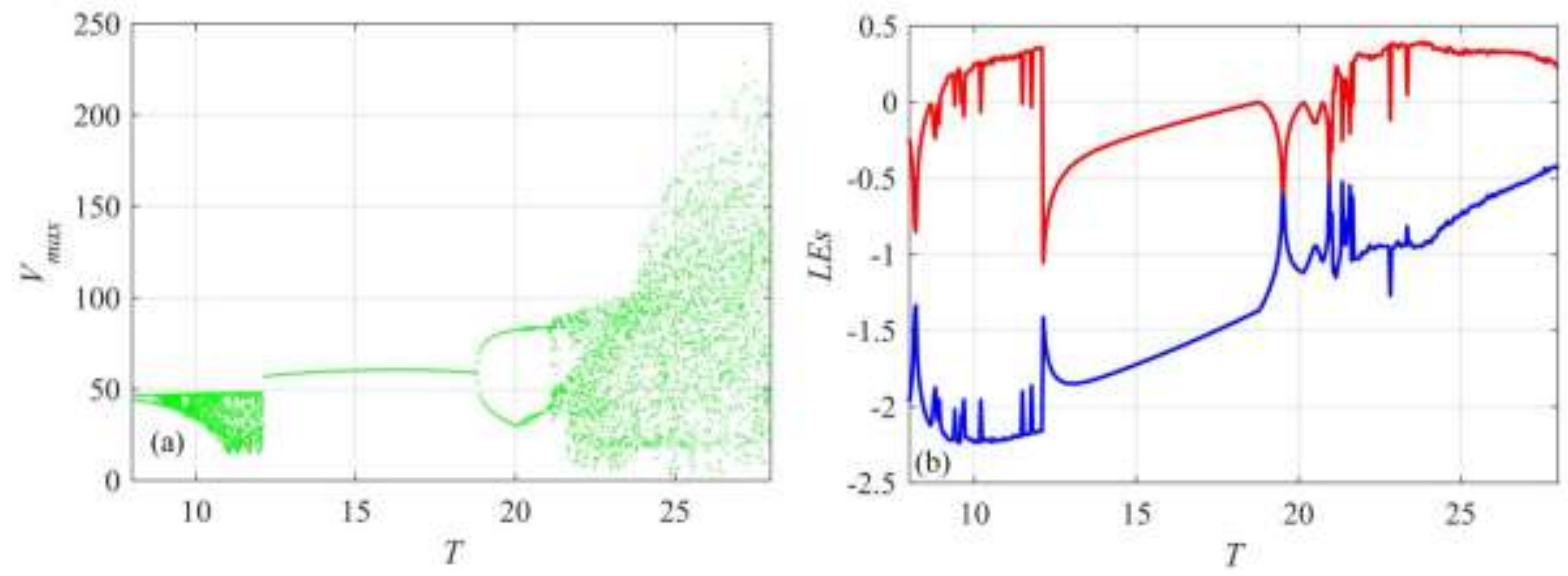

Fig. 4: Dynamics of the system with the variation of parameter T. (a) Bifurcation diagram; (b) Lyapunov exponents.

Secondly, the Lyapunov exponents based chaos diagrams are obtained in different parameter planes. Let $g_{l}=0.1$, T varies from 8 to 28 with step size of 0.1 and $g_{d}$ varies from 1 to 2 with step size of 0.005. The analysis result in the parameter plane T-gd is shown in Fig.5 (a). Let $g_{d}=1.5$, T varies from 8 to 28 with step size of 0.1 and $g_{l}$ varies from 0 to 1.5 with step size of 0.0075 . The analysis result in the parameter plane $T-g_{l}$ is shown in Fig.5 (b). Let $\mathrm{T}=11, g_{d}$ varies from 1 to 2 with step size of 0.005 and $g_{l}$ varies from 0 to 1.5 with step size of 0.0075 . The analysis result in the parameter plane $T-g_{d}$ is shown in Fig.5 (c). As shown in those chaos diagrams, the system has wide regions for chaos. Meanwhile, there are also regions with Lyapunov exponents smaller than zero, and the white regions mean that the system is divergent. It shows that the system has rich dynamics with the variation of parameters $g_{l}, g_{d}$ and $\mathrm{T}$. 
(a)

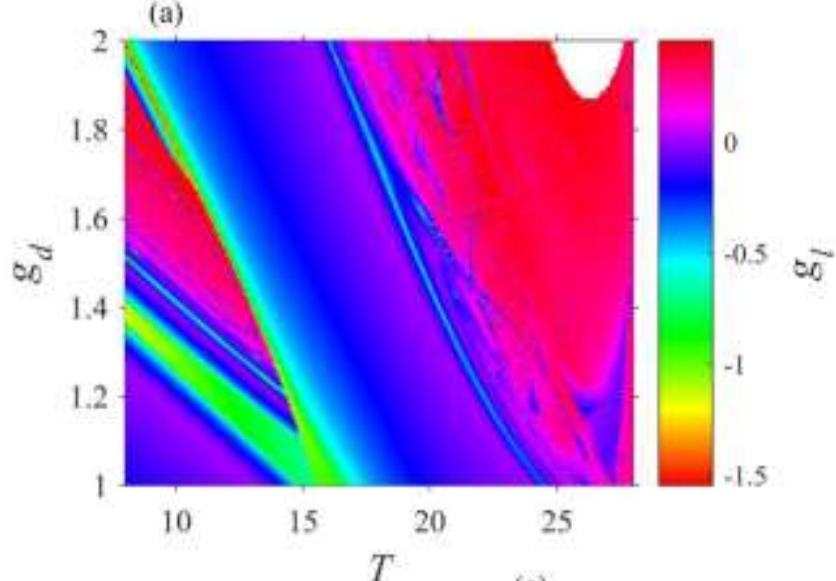

(b)

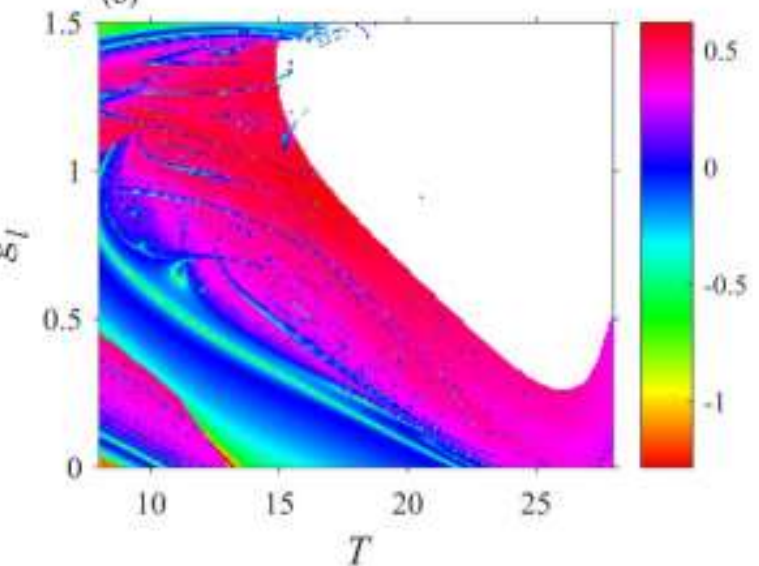

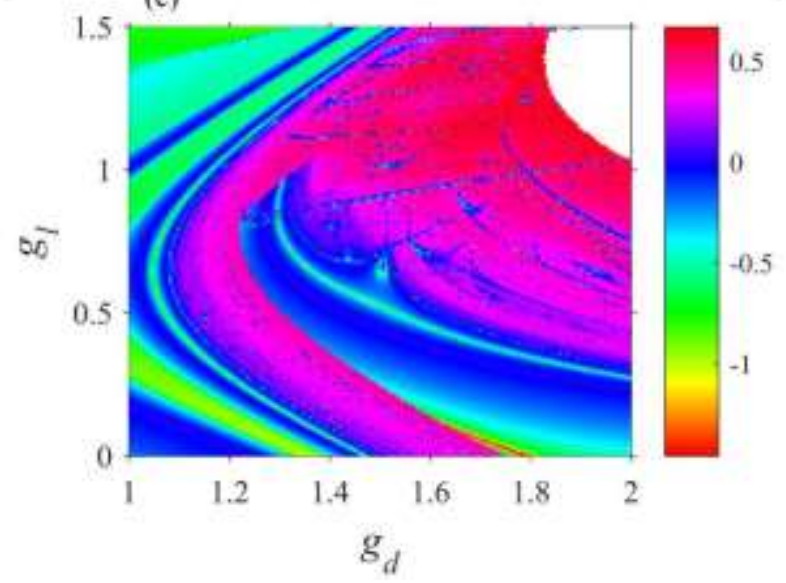

Fig. 5: Lyapunov exponents-based chaos diagrams in different parameter planes. (a) $T-g_{d}$; (b) $T-g_{l}$; (c)

$$
g_{d}-g_{l}
$$

\subsection{Complexity measure}

In this paper, complexity measure of time series generated by the neural is carried out based on the sample entropy (SampEn) algorithm. For a given time series $\{x(n), n=0,1,2, \ldots, N-1\}$, its SampEn is estimated by the following steps.

Step 1: For a given integer number $\mathrm{m}(m \leq N-2)$, the reconstructed phase-space is denoted as

$$
X(i)=[x(i), x(i+1), \mathrm{L}, x(i+m-1)]
$$

where $i=1,2, \ldots, N-m+1$.

Step 2: Define the distance as

$$
d[X(i), X(j)]=\max _{k=0, \mathrm{~L}, m-1}\{|x(i+k)-x(j+k)|\}
$$

where, $i, j=1,2, \ldots, N-m+1$ and $\mathrm{i} \neq \mathrm{j}$. 

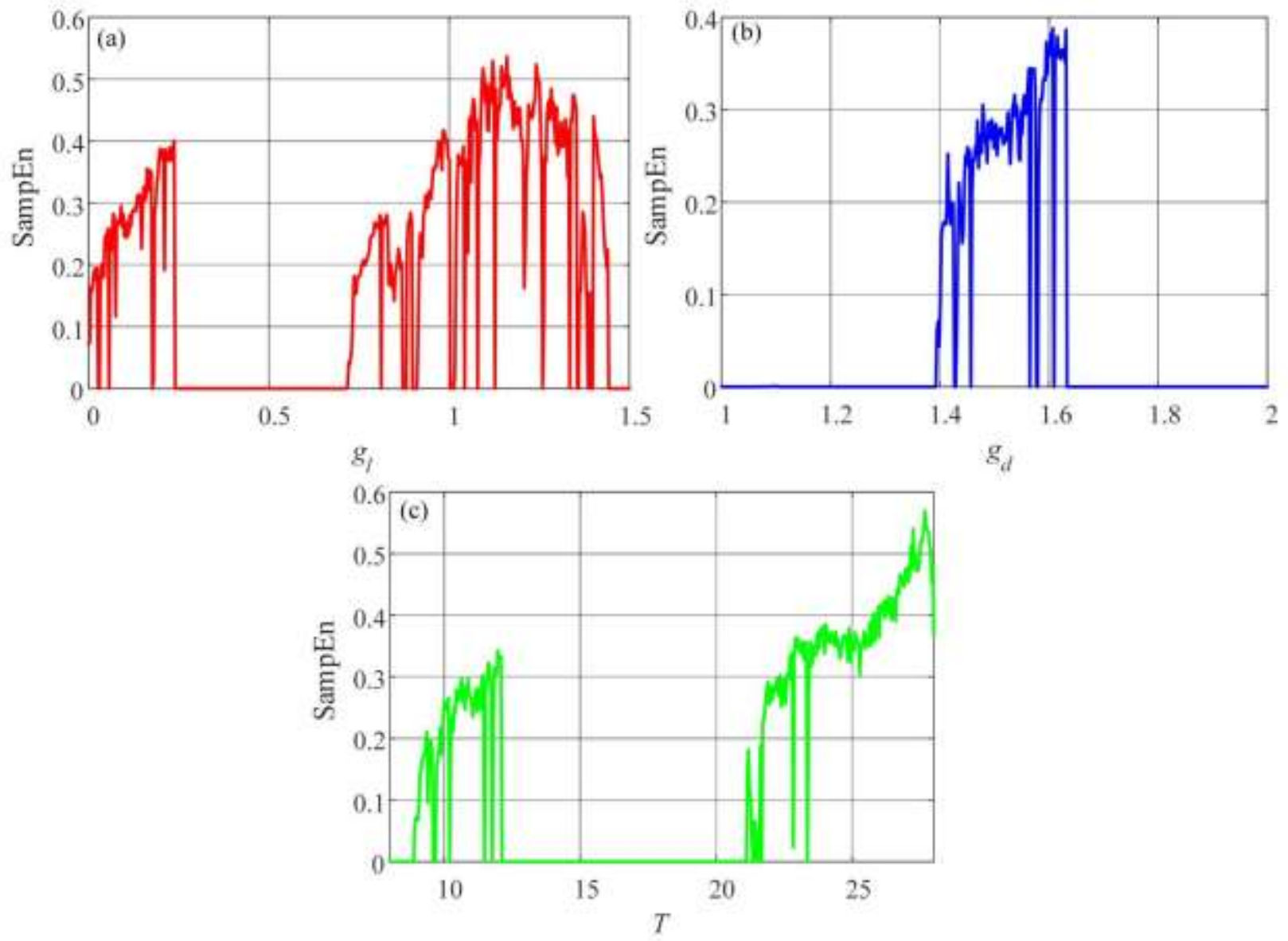

Fig. 6: SampEn complexity analysis results with different parameters. (a) gl varying; (b) gd varying; (c) T varying

Step 3: For a given threshold value $r$,

$$
C_{i}^{m}(r)=\frac{1}{N-m+1} \#\{d[X(i), X(j)]<r\}
$$

where \# denotes number, and $i=1,2, \ldots, N-m+1$ and $\mathrm{i} \neq \mathrm{j}$.

Step 4: Define

$$
\Phi^{m}(r)=\frac{1}{N-m+1} \sum_{i=1}^{N-m+1} C_{i}^{m}(r)
$$

Step 5: Increase the dimension $m$ as 1 and repeat the above four steps. Then we can get $\Phi^{m+1}(r)$, and the SampEn is estimated by

$$
\operatorname{SampEn}(x, m, r)=-\ln \frac{\Phi^{m+1}(r)}{\Phi^{m}(r)}
$$


Obviously, there two parameters for this algorithm. One is phase space dimension $m$ and the other is the similar tolerance $r$.
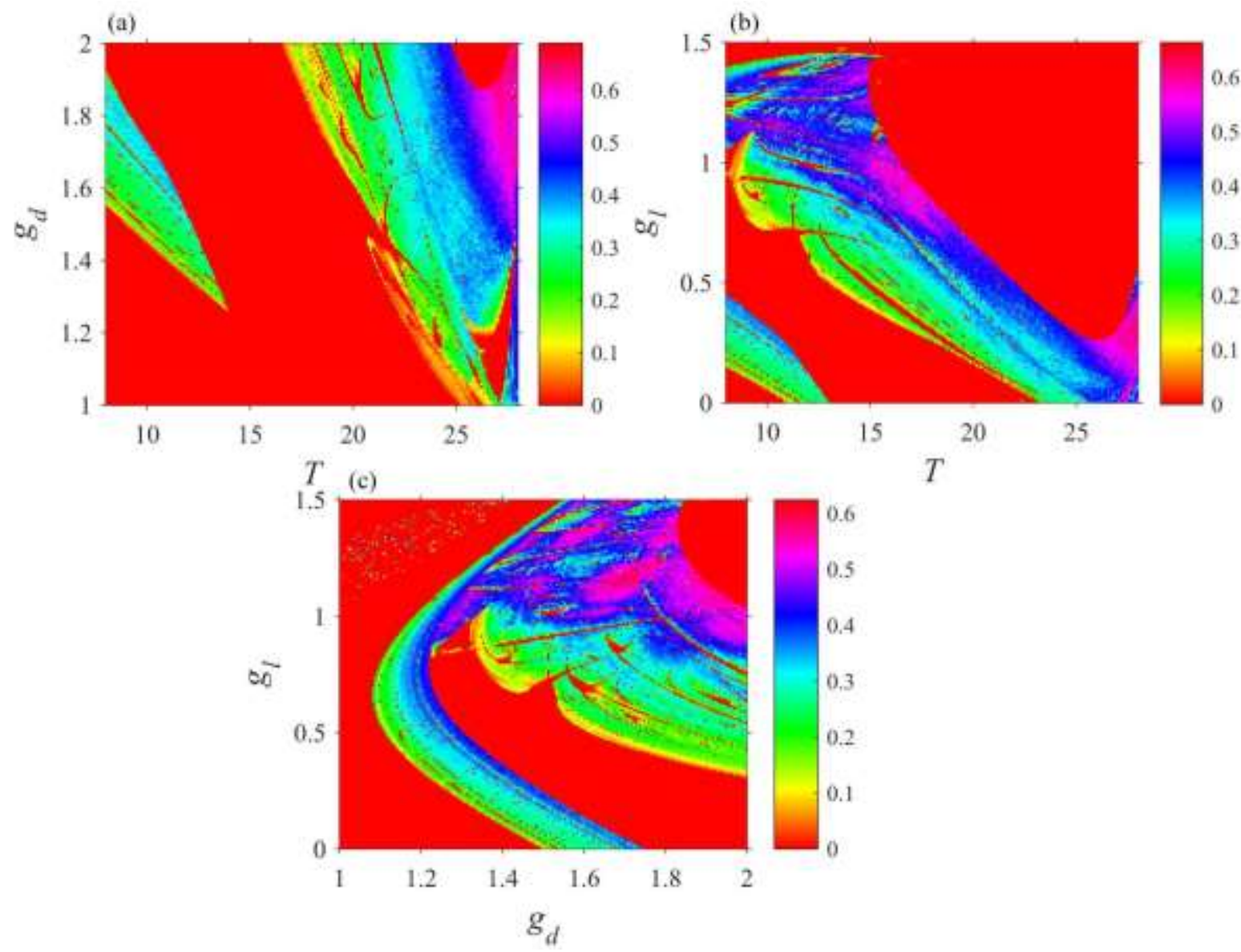

Fig.7: SampEn based chaos diagrams in different parameter planes. (a) T-gd; (b) T-gl; (c) gd-gl

In general, the larger $\mathrm{M}$ allows a more detailed reconstruction of the dynamic evolution of the system. The value of similar tolerance $r$ is a issue worth considering. Too small similar tolerance will lead to the loss of information. In the practical applications, we defines $r=r * S D$, where SD (the standard deviation) is the standard deviation of the original one dimensional time series. In this paper, $m=3$ and $r=0.15$ saying $r=0.15 \mathrm{SD}$. The physical significance of SampEn is not difficulty to find out. It measures the probability of the generation of new patterns in the time series. The larger the measure value is, the greater the probability of the generation of new patterns is, that is, the higher the sequence complexity is.

Complexity of the system with the variation of different parameters are shown in Fig.6. The parameters and initial conditions are the same as the corresponding dynamics part as shown in Figs.3(a), 3(b)-2 and 3(c). Meanwhile, time series V is used to estimate the complexity with first 1000 points of data removed. The length of each time series is 9000 . Obviously, the curves of complexity agree well with the dynamical analysis results. Complexity analysis results show high complexity with chaotic state while show low complexity and zero complexity for the other states. Obviously, for those periodic windows, there shows low complexity measure results. When gd 
takes values between 1.4 and 1.63, the complexity increase with the increase of gd, and when $\mathrm{T}>21.1$, the complexity of the system increases with the increase of $\mathrm{T}$. However, compared with dynamics analysis results, SampEn can build a clearer version regarding the complexity of the system. Thus we analyzed complexity of the neural model in the parameter planes. As with Fig.5, we use the same parameter settings and initial conditions. It shows in Fig.7 that the high complexity region in those chaos diagrams match well with the chaotic region as shown in Fig.6. According to the above analysis results, it shows that the neural system can also have high complexity measures which is related to the chaotic state, and SampEn provides an effective tool for complex dynamics of chaotic systems.

\section{Spiral Waves in the DHBN model}

The major challenge of a map-based neuron model is to correlate the behaviour with a biophysical neuron characteristic. Replicating the network behaviour of a continuous time neuron model with a map-based neuron is considered complex as phenomenon like the spiral waves and multiarmed spiral waves are not very well documented by literatures [46]. Hence a discrete neuron model capable of replicating the behaviour of an ODE neuron model in both local and collective sense is very rarely discussed. The DHBN model proposed in this work has effectively showed the characteristics of a biophysical neuron [47] and we now are interested in investigating the network behaviour of the DHBN model.

For this we arrange the neuron in a network and depending on the number of layers in the network we subdivide the discussion in to two cases. In the first case we investigate using a single lattice array of DHBN model and in the second case we consider two-layer network with each layer being the lattice array of the DHBN model. Further we expand the investigation by considering two separate scenarios which could affect the excitability of the neurons and thus could contribute to re-entry and spiral waves. In the first scenario we considered different sizes and orientations of obstacles and show their impact on the wave propagation and re-entry. The mathematical model of the DHBN network can be defined as

$$
\begin{aligned}
C V_{i j}^{k+1}= & -I_{L_{i j}}^{k}-I_{D_{i j}}^{k}-I_{R_{i j}}^{k}-I_{S D_{i j}}^{k}-I_{S R_{i j}}^{k} \\
& +\sigma\left(V_{i+1 j}^{k}+V_{i-1, j}^{k}+V_{i, j+1}^{k}+V_{i, j-1}^{k}-4 V_{i j}^{k}\right)+A \sin (\omega t) \theta_{i \tau} \theta_{j \rho}
\end{aligned}
$$

The stimuli is applied to the network with the condition $\theta_{i \tau}, \theta_{j \rho}=1$ when $i=\tau, j=\rho$. The stimulus is applied from the left side of the network by setting $\tau=100, \rho=1$. The stimuli settings are $A=0.1, \omega=0.01$ and we capture the spatiotemporal behaviour of the network at the end of 6000 s.

When the stimuli is applied to the network, the tissues are highly excited and we could note simple travelling waves from left to right boundary and no signs of wave re-entry. This can be seen from Fig.8 and varying the stimulus amplitude, frequency and the system parameters doesn't change the excitability of the neurons. For certain amplitude and frequency values of the stimulus the entire network goes unstable. Hence we are interested in investigations different scenarios which will influence the wave re-entry and spiral waves in the network. 


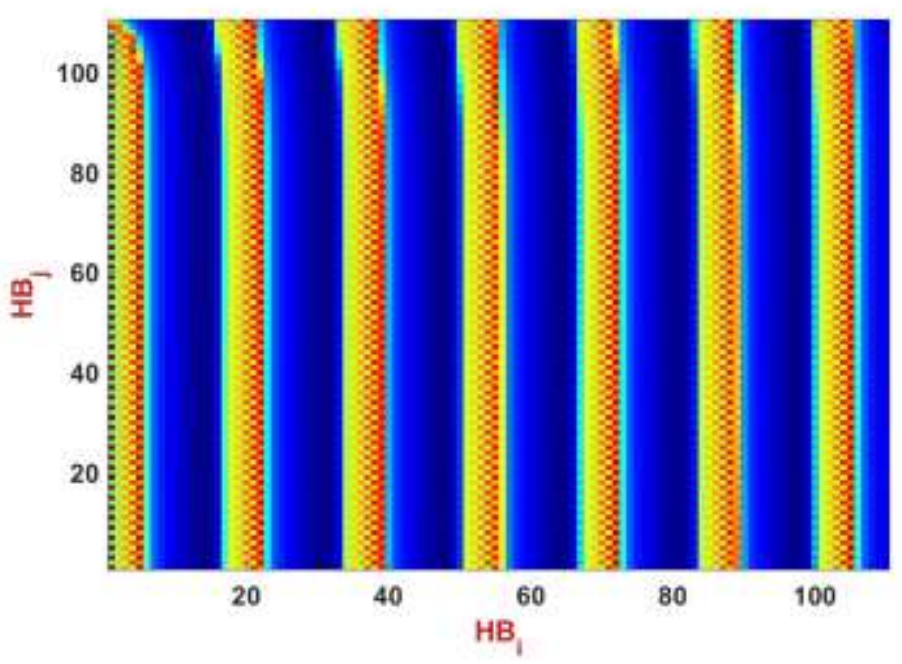

Fig.8: Travelling waves seen in the DHBN network excited by a periodic stimuli. The amplitude and frequency of the stimuli are 0.1 and 0.01 respectively. The coupling strength $\sigma=1$ and we considered no flux boundary conditions for the network.

In our previous work [44], we have showed that obstacles in the network can initiate wave re-entry and can contribute to the spiral wave formations. Interestingly literatures dealt with the effects of noise on the spiral waves in the network. Hence, we wish to study the impact of obstacles or noise in wave re-entry phenomenon. In scenario-A we consider that the network has some defects defined by the obstacles on the path of the wave propagation and in scenarios-B we consider Noise effects in the discussion and study both spiral wave birth and death because of noise. In scenarioB we also investigate the combined effects of both obstacle and noise.

\subsection{Effects of obstacle on the spatiotemporal behaviour}

We have shown that in the absence of obstacles the network tissues are in high excitability and hence plane waves propagate in the media (Fig.8). Now we insert an obstacle in the network and investigate the effect of the size and orientation of the obstacle on the wave front. We consider the system parameters as in (5) and the stimulus setting are same as used in Fig.8. For the orientations of the obstacle we have considered parallel and perpendicular obstacles with reference to the wave front. Different sizes of obstacles having heights $H \in[60,20]$ and width $W \in[20,10,5,1]$ with the measuring unit being the nodes. First we considered parallel obstacles with different combinations of $\mathrm{H}$ and $\mathrm{W}$ as shown in Fig.9. When the obstacle width is less than 5 nodes, there is no wave reentry in the media and we could note just a phase shift in the wave propagation as in Fig.9(a) for the $[H, W]=\{60,1\}$. When the width is increased to 5 and keeping the height as 60 , the obstacle creates wave re-entry and spiral wave seeds in the right boundary of the obstacle. Further increasing the width to 10 two spiral pools are created at the top and bottom ends of the obstacle and we could also note that the spiral waves are formed inside the obstacle too. Broken multiple spiral waves can be generated by further increasing the width to 20 as in Fig.9(d). Now we decrease the height to 1 and width to 5 and no phenomenal changes in the excitability of the neurons and hence travelling plane waves are noted. Now an increase in just the width of the obstacle to 10 making it a square block could induce wave re-entry and spiral waves shown in Fig.9(f). 
a

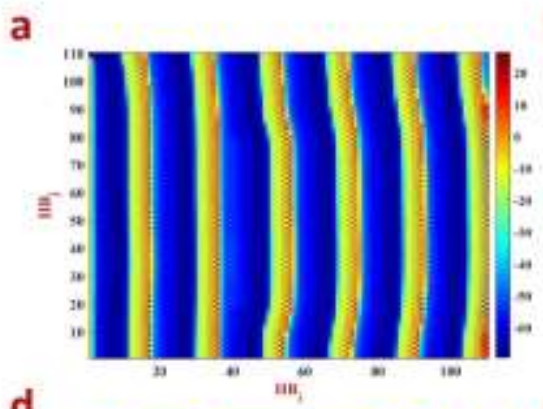

d

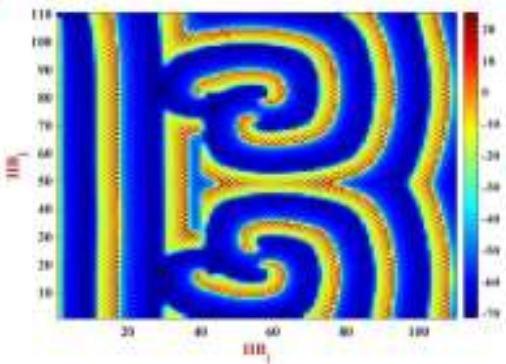

b

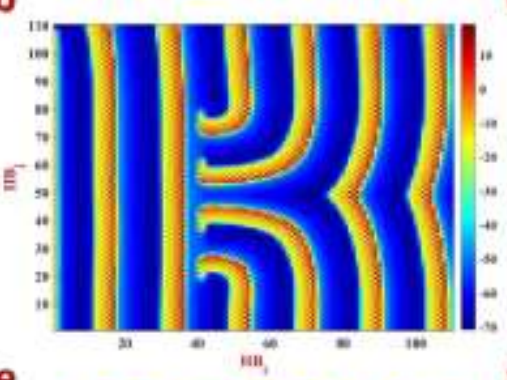

e

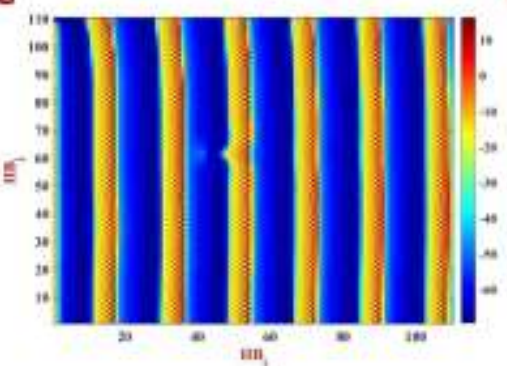

c

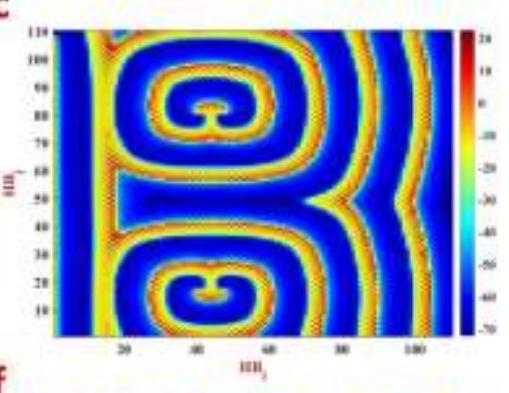

f

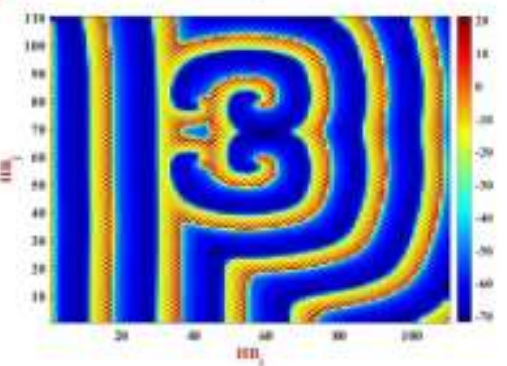

Fig.9: Spatiotemporal behaviour of the DHBN network considering the obstacle parallel to the wave front. Different sized obstacles are used for the analysis with obstacles greater than five node width we could note that wave re-entry and spiral waves are formed near the boundary opposite to the wave entry side.

Considering the obstacles placed perpendicular to the wave front, we considered different sizes of obstacles with width $\mathrm{W} \in\{20,10,5\}$ and length $L \in\{60,20,10,5\}$. Firstly, we consider an obstacle of 10x5 and the excitation is applied to the network. The snapshots shown in Fig.10a confirms that the obstacle breaks the wave at the interaction points while no re-entry or spiral wave phenomenon observed. By increasing the width to 20 and length kept at 10, the spiral wave seeds are seen towards the upper and lower part of the obstacle as in Fig.10(c). For an obstacle of width 20 and length 60 , symmetric spiral waves originate at the right side of the obstacle and rotates with a larger arm radius. But comparing Fig.9 with 10, we could comment that obstacles parallel to the wave front are more effective in the excitability and wave re-entry compared to the perpendicular obstacles. 

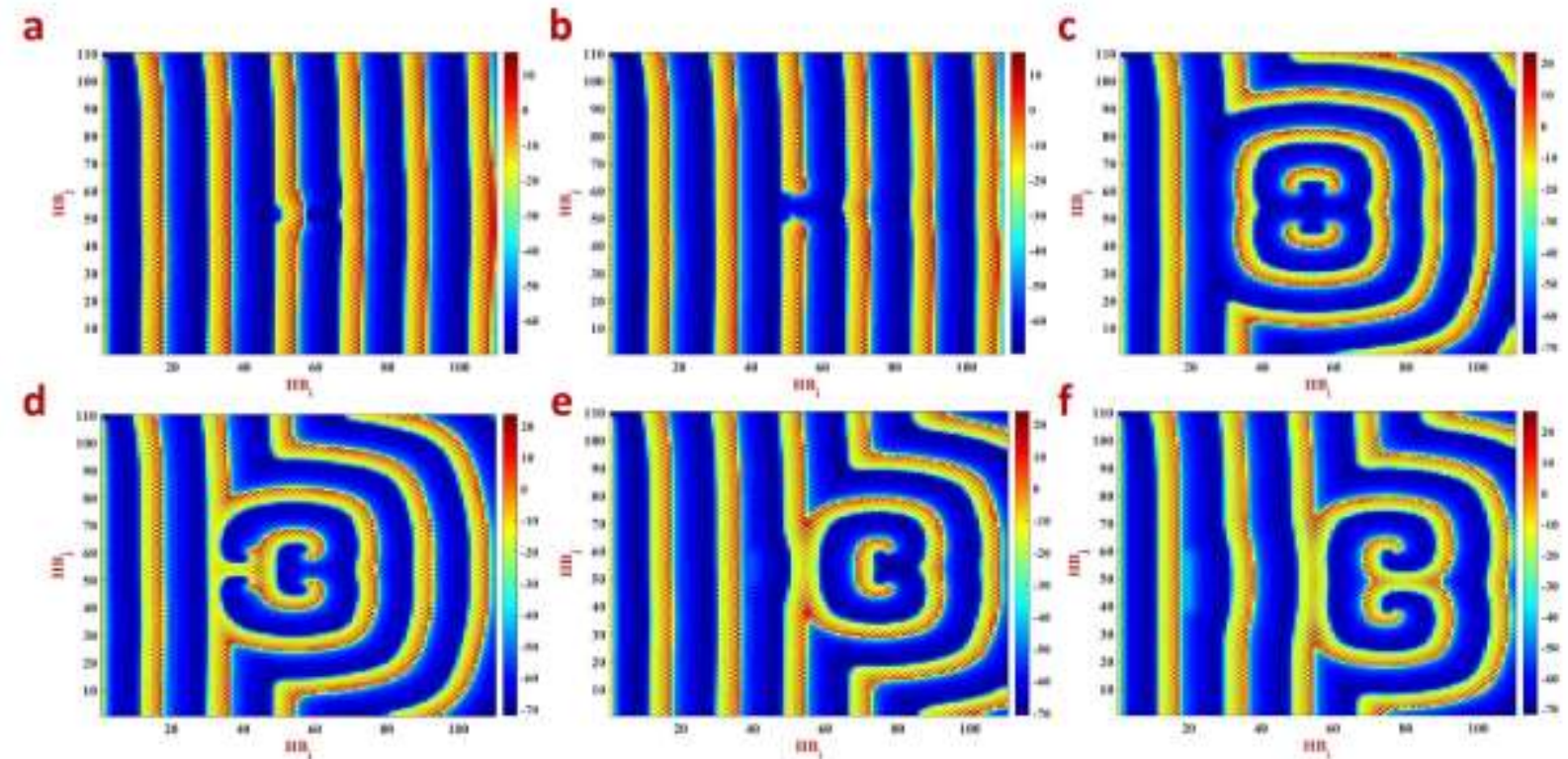

Fig.10: Snapshots of the media with obstacle perpendicular to the wave entry. Different combinations of width and length of the obstacle are considered with system parameters and stimuli settings same as used in Fig.8.

Now the question arises of what will be affect of obstacles in multilayer networks. Will the obstacle in a layer influence the excitability in the other layers too? If so under what circumstances such excitability changes happens. To answer all these questions, we construct the mathematical model of the two-layer DHBN network as in (8)

$$
\begin{aligned}
C V_{U, i j}^{k+1}= & -I_{L_{U, i j}}^{k}-I_{D_{U, i j}}^{k}-I_{R_{U, i j}}^{k}-I_{S D_{U, i j}}^{k}-I_{S R_{U, i j}}^{k}+D_{L U}\left(V_{\mathrm{L}, i j}-V_{\mathrm{U}, i j}\right) \\
& +\sigma_{U}\left(V_{U, i+1 j}^{k}+V_{U, i-1, j}^{k}+V_{U, i, j+1}^{k}+V_{U, i, j-1}^{k}-4 V_{U, i j}^{k}\right)+A_{U} \sin (\omega t) \theta_{i \tau} \theta_{j \rho} \\
C V_{\mathrm{L}, i j}^{k+1}= & -I_{L_{\mathrm{L}, i j}}^{k}-I_{D_{\mathrm{L}, i j}}^{k}-I_{R_{\mathrm{L}, i j}}^{k}-I_{S D_{\mathrm{L}, i j}}^{k}-I_{S R_{\mathrm{L}, i j}}^{k}+D_{21}\left(V_{\mathrm{U}, i j}-V_{\mathrm{L}, i j}\right) \\
& +\sigma_{\mathrm{L}}\left(V_{\mathrm{L}, i+1 j}^{k}+V_{\mathrm{L}, i-1, j}^{k}+V_{\mathrm{L}, i, j+1}^{k}+V_{\mathrm{L}, i, j-1}^{k}-4 V_{\mathrm{L}, i j}^{k}\right)+A_{L} \sin (\omega t) \theta_{i \tau} \theta_{j \rho}
\end{aligned}
$$

The inter layer coupling term is defined by $D_{L U}\left(V_{\mathrm{L}, i j}-V_{\mathrm{U}, i j}\right)$ for the upper layer and $D_{21}\left(V_{\mathrm{U}, i j}-V_{\mathrm{L}, i j}\right)$ for the lower layer. The intra layer coupling is like the one used in single layer network (7) with $\sigma_{U}, \sigma_{L}$ defining the respective coupling strengths. We have considered stimulus whose amplitudes are $A_{U}$ and $A_{L}$ for upper and lower layers respectively and by setting the amplitude of the stimulus in the respective layer to ' 0 ' we could show the effect of coupling effects between layers.

In the first discussion we consider the obstacles to be parallel to the wave front and stimulus is applied only to the upper layer by setting $A_{L}=0$. We have considered similar sizes of obstacles as considered for the single layer discussion and the obstacle is inserted only in the upper layer. Though there is no obstacle in the lower layer, the excitability of tissues is controlled by the first layer through the coupling as there is no stimulus in the lower layer. Hence, we could note that both the layers operate in synchrony and the obstacle effects seen in the upper layer is transferred 
to the lower layer and thus both the layers showing similar re-entry and spiral wave phenomenon. These observations can be easily seen in the snapshots shown in Fig.11. There is an identifiable difference between the upper layer and lower layer snapshots with the lower layer displaying a much lesser difference between the media and excited tissues while the upper layer shows a definitive larger difference. This is because the excited state of neurons in the lower layer is of lesser amplitude as the coupling though is capable of achieving coherent behaviour between the layers, they are not effective in coupling the exact excitability conditions to the lower layer.
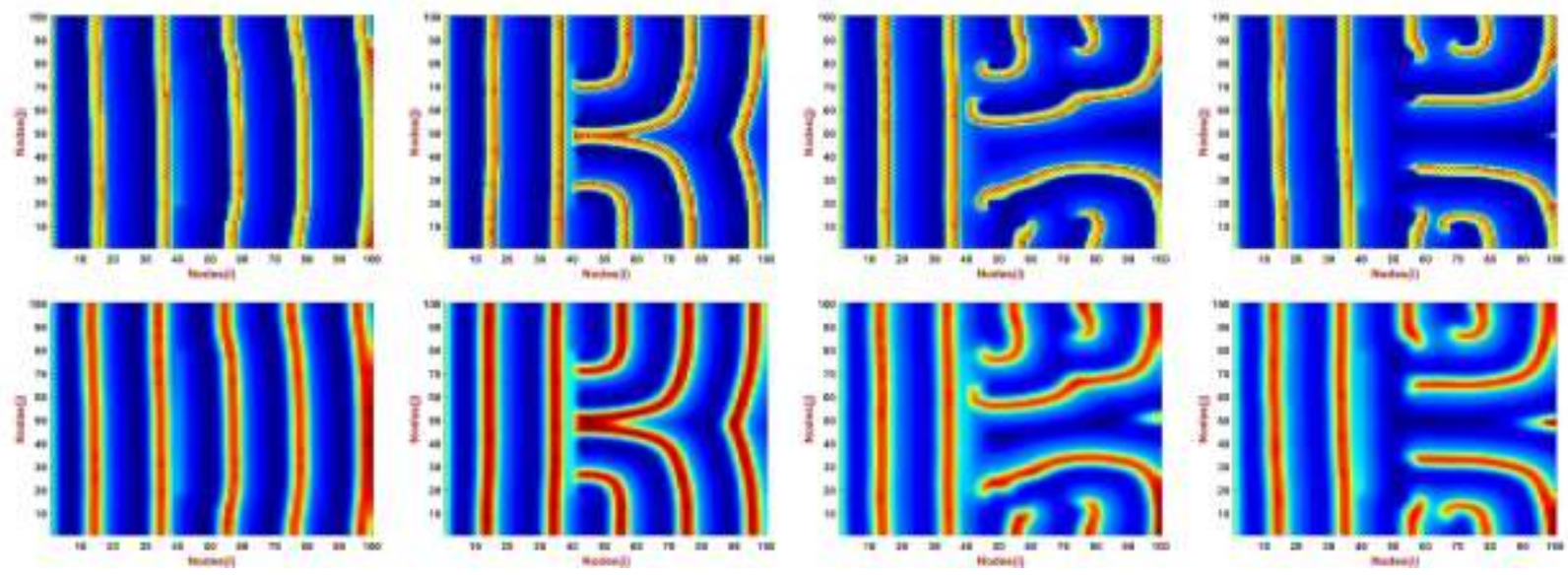

Fig.11: Spatiotemporal snapshots of the two-layer DHBN network with the obstacle and stimulus applied to the upper layer while the lower layer is coupled to the upper layer with a coupling strength of 0.05 .

When stimulus is applied to only upper layer the obstacle effects are coupled to the lower layer even though there is no physical obstacle present. Now we consider stimulus in both the layers and use the same orientation and size of obstacle as used in Fig.11. Now we could show that though the coupling strength or the stimulus amplitude is note changed as from Fig.11, the obstacle effects in the lower layer are not displayed. In other words, now the lower layer tissues are excited by the applied stimulus rather than the upper layer effects as shown in Fig.12. Thus, the effects of coupling bond between the layers are now eliminated by applying stimulus to the respective layers. Also we could note that the difference between the excited amplitude and the media in the lower layer are significantly higher and thus overcoming the blur effect seen in Fig.11. Though not presented here, the same phenomenon can be observed when we consider the lower layer obstacle and excitation with the upper layer not exposed to obstacles or stimulus. 

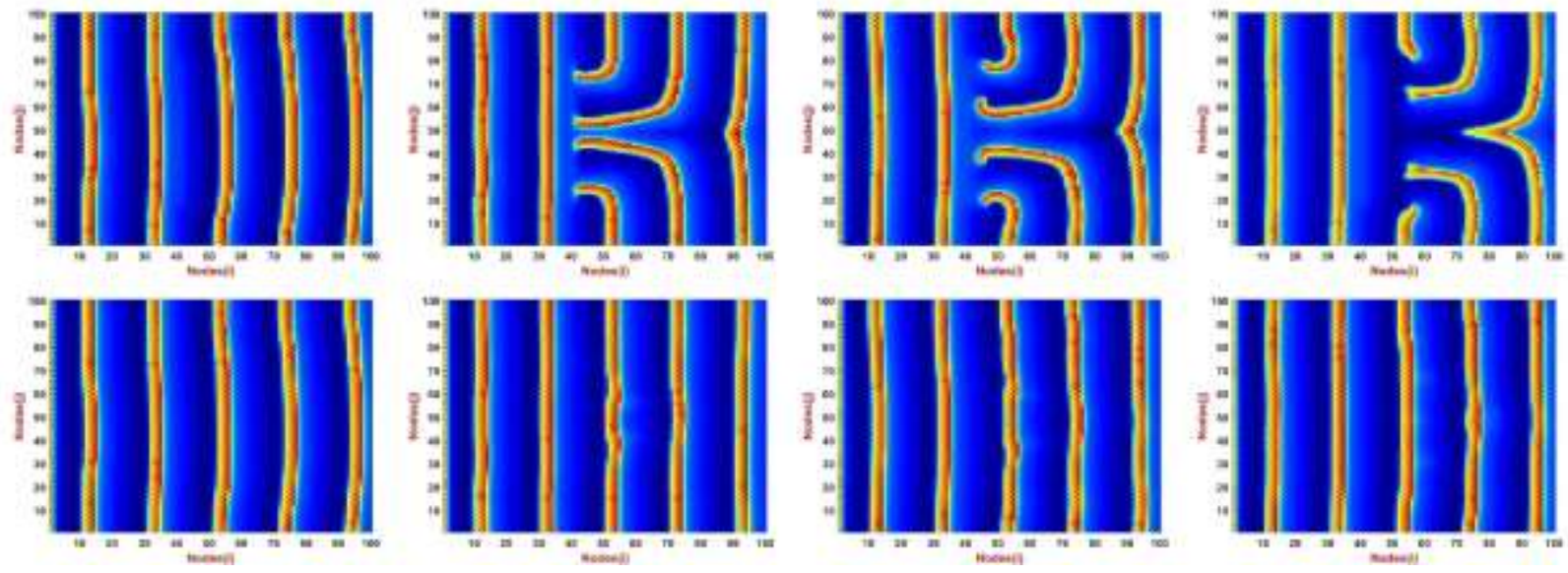

Fig.12: The snapshots of the two-layer network with stimulus applied to both the layers while the obstacle is considered only in the upper layer. The stimulus and coupling strength settings are similar to Fig.11.

\subsection{Effects of noise on the spatiotemporal behaviour}

In this section we consider a stochastic noise which will be added to the DHBN model as an additional current term. Under the influence of noise, we investigate the spatiotemporal dynamics of the DHBN network without and with obstacles. The stochastic noise used in the investigation is the Box-Mueller type noise[14, 23, 48, 49] defined by

$$
N_{k}=\cos (2 \pi \alpha) \sqrt{(-4 \delta \ln (\beta))}
$$

The parameters $\alpha, \beta$ are randomly chosen between $[0,1]$ and $\delta$ is the noise variance. The noise variance is considered as the control parameter in the discussions in this section.

\subsubsection{Effect of noise in single layer network:}

We consider the single layer network discussed in (7) and add the noise term (13) and the parameters and the stimulus settings are same as used in Fig.8. Firstly, we consider no obstacles in the network and under different noise variances we investigate the wave propagation and excitability of the neurons. Interestingly when $\delta=0$ (Fig.8) the neurons are in highly excited state and show no sign of spiral waves which was the case for $\delta=1$ too. But for $\delta=1$, a phase change is seen in the wave propagation which shows signs of re-entry and hence we increased to $\delta=3$. Now around the node [60.80] a simple re-entry is seen and while increasing the simulation time to 10000 s we could note much change in the spiral seed. But for $\delta=5$ the excitability of most of the tissues are disturbed resulting in multiple broken spiral waves which are further divided into smaller spiral seeds for $\delta=7$. Finally, for a noise variance $\delta \geq 10$ the spiral waves are eliminated and most of the neurons enter idle state. Thus, without obstacles we could observe wave re-entry and spiral wave formations in the DHBN network, and this is because of the noise term changing the excitability of the tissues. 

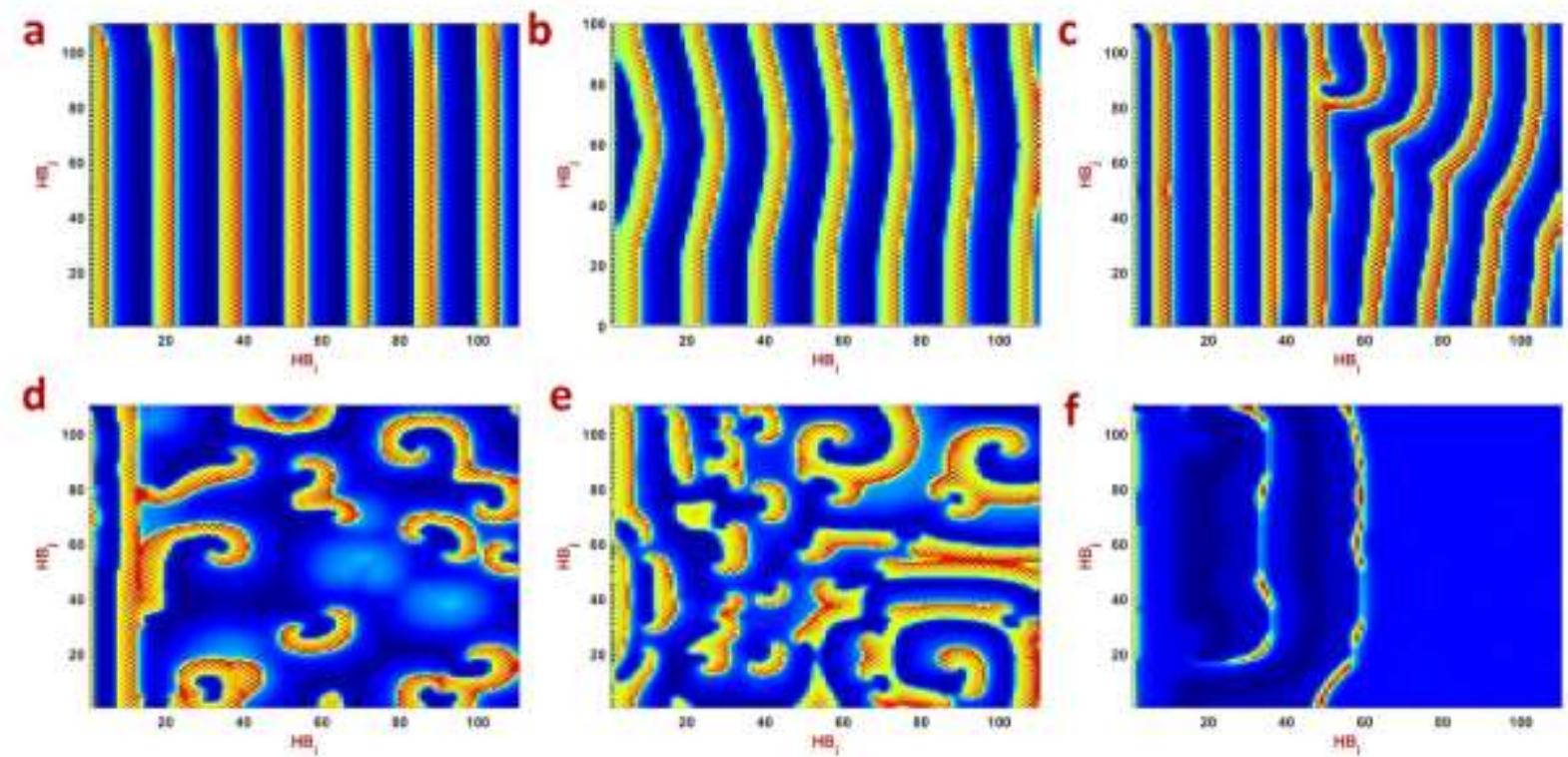

Fig.13: Spatiotemporal snapshots of the single layer network without obstacles for different noise variances $[0,1,3,5,7,10]$ displayed with figure labels $[\mathrm{a}, \mathrm{b}, \mathrm{c}, \mathrm{d}, \mathrm{e}, \mathrm{f}]$ respectively.

Though we have shown that without obstacles, the DHBN network shows wave re-entry and spiral waves in the presence of noise, we are interested in studying the combined effect of obstacles and noise. Hence, we introduce a vertical (parallel) obstacle as used in Fig.9d and consider different noise variances to investigate the combined effect. For any $\delta<1$, the noise doesn't have effect on the spatiotemporal behaviour of the network but $\delta=1$, the spiral waves are pushed to the right boundary because of the excitability change in the neurons. The network soon show signs of plane waves in some regions near the left boundary while the right half still show multiple broken spiral seeds for $\delta=5$. Further increasing $\delta$ results in the multiple re-entry and spiral waves due to the larger change in the neuron excitability because of hight noise variance. For $\delta=12$ most of the tissues enter idle state while the tissues near the left boundary has some re-entry phenomenon. The right boundary shows all the nodes locked to an high spike voltage with no resetting. This is because of the noise variance disturbing the reset condition due to the higher noise variance. 
a

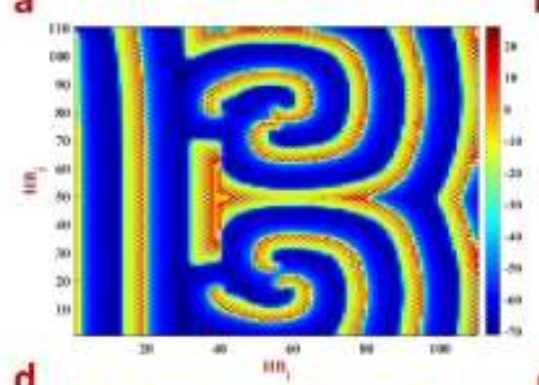

d

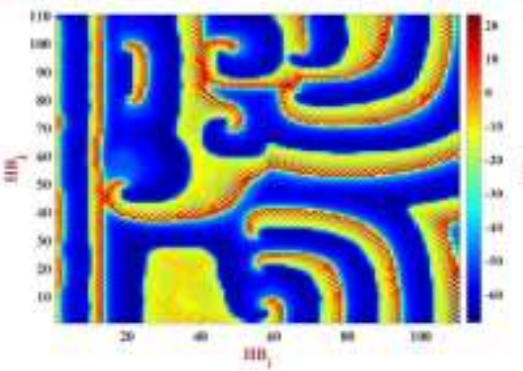

b
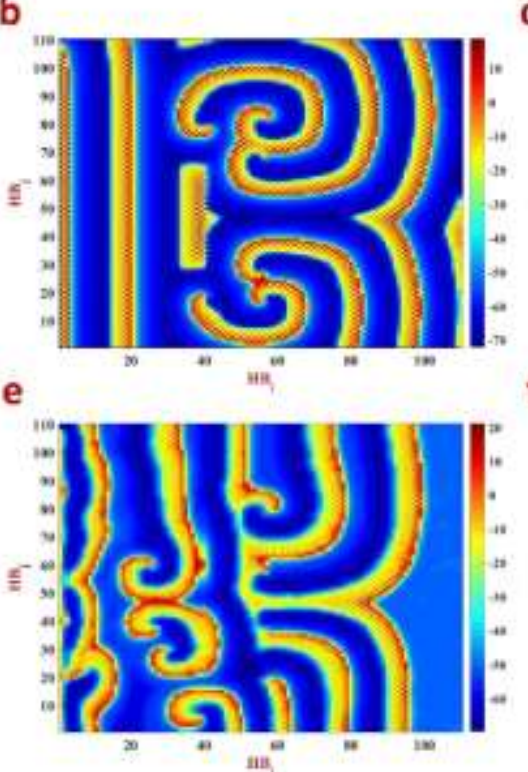

C

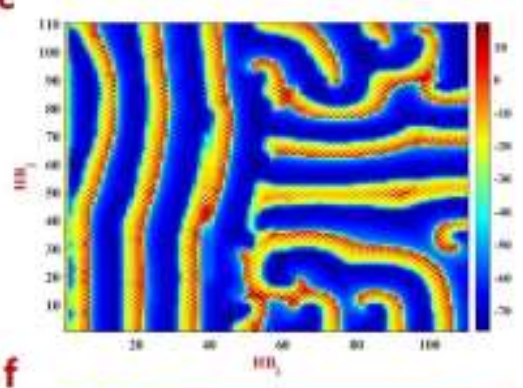

$\mathrm{f}$

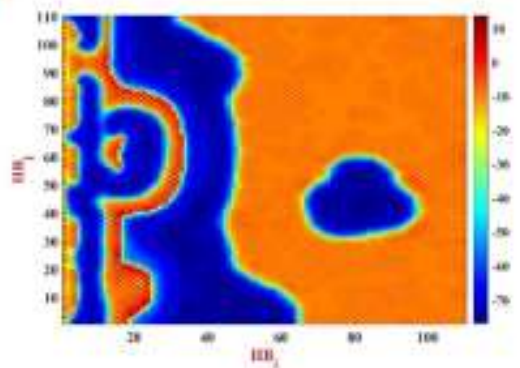

Fig.14: Spatiotemporal snapshots of the single layer network with obstacle as used in Fig.9d for different noise variances $[0.5,1,5,7,10,12]$ displayed with figure labels $[a, b, c, d, e, f]$ respectively. The obstacle is placed parallel to the wave entry.

Similar behaviour is seen when we changed the obstacle perpendicular to the wave entry as in Fig.10e. Though for lesser noise variance $\delta<3$ there is no much definable change in the spiral waves, for $\delta=5$, the network divides in to two half vertically with the left half showing plane waves with nodal phase shifts and the right half showing spiral seeds. This is because the noise variance overcomes the effect of the stimulus when it crosses from left to right and thus changing the excitability of the tissues in the right half. But for $\delta=7$ the network display multiple spiral waves with pools comprising neurons locked to high spike voltages. The spiral waves are soon dissipated with only few spiral seeds seen in the network for $\delta=9$. Comparing Fig.14 and Fig.15 we could confirm that obstacles placed parallel to the wave front shows more complete effects for noise compared to the obstacle placed perpendicular to the wave front. 

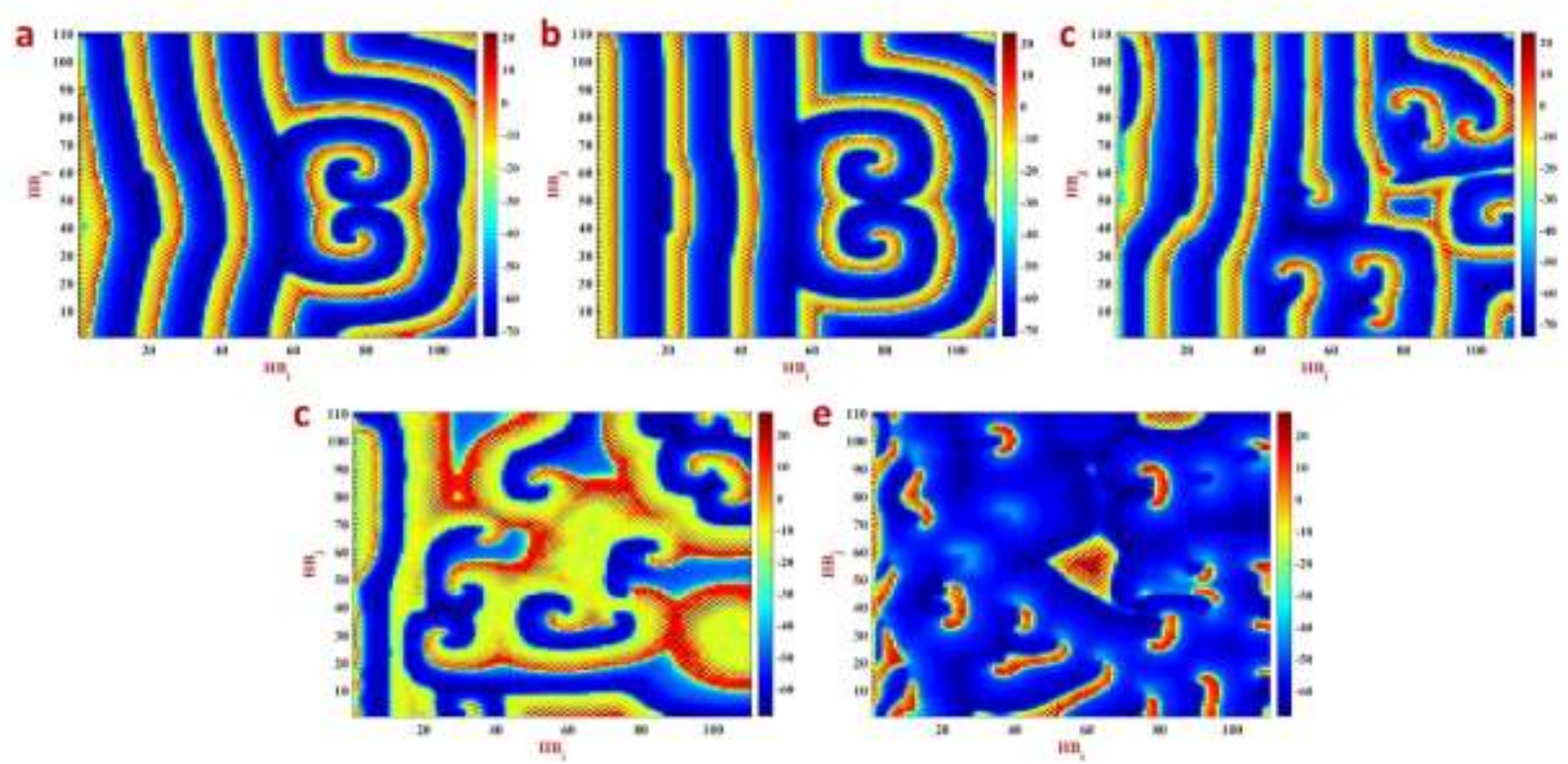

Fig.15: Spatiotemporal snapshots of the single layer network with obstacle as used in Fig.9d for different noise variances $[0.5,1,5,7,9]$ displayed with figure labels $[\mathrm{a}, \mathrm{b}, \mathrm{c}, \mathrm{d}, \mathrm{e}]$ respectively. The obstacle is placed perpendicular to the wave entry.

\subsubsection{Effect of noise in two-layer network:}

Now we include noise in the two-layer network (8) and the noise effects are considered in both the layers with stimulus applied to the layers at an amplitude of 0.01 and frequency of 0.1. Firstly, we consider that there is no obstacle in both the layers and the stimulus is applied form the left boundary. When the noise variance $\delta \leq 1$, the neurons are in high excitability and thus the network shows simple plane wave propagation as in Fig.16(a). Increasing the noise variance to $\delta=5$, we could observe spiral waves formed in many regions of the network showing that the noise induced current in the nodes make their excitability vary and thus resulting in wave re-entry and spiral waves. When the noise variance $\delta$ is further increased to 8 , due to the stochastic nature of the noise current, both the layer show behaviour which confirms the break in their coupling due to the noise randomness. Also, the first layer shows signs of entering state of high excitability with very low frequency of excitability resetting whereas the lower layer still shows wave re-entry and spiral waves. Further increase in $\delta$ to 10 , most of the nodes enter into an idle state resulting in small pools of idle nodes near the right boundary of the network. Due to the applied stimulus, both the layers show spiral waves in the left half of the network which soon dissipates as the wave crosses to the right half. 

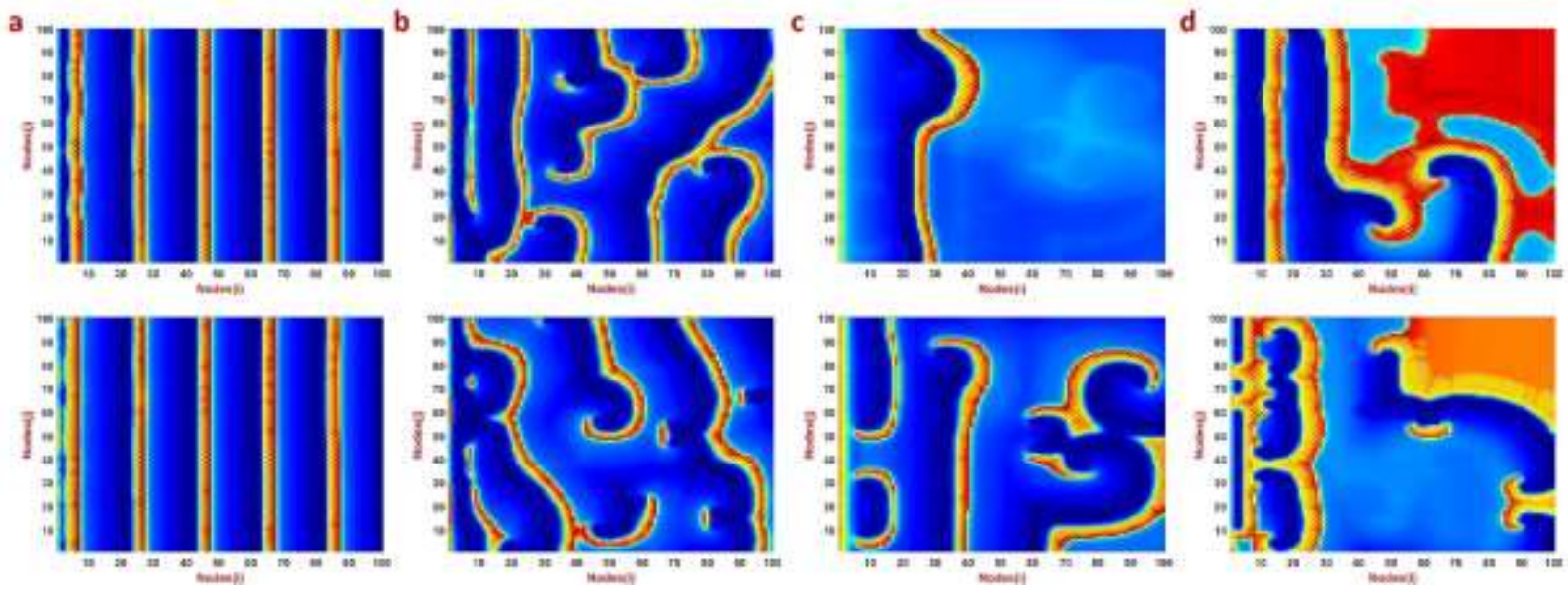

Fig.16: Spatiotemporal behaviour of the two-layer DHBN network considering noise presence in both the layers while there is no obstacle considered. The stimulus is applied to both the layers and the sub figures $[a, b, c, d]$ shows the snapshots of the two layers for various values of the noise variance $[1,5,8,10]$ respectively.

Now two different sizes and orientations of obstacles are places in the layer and we investigate the effect of noise. In the first layer we placed an obstacle parallel to the wave entry and in the second layer we placed a perpendicular obstacle. Fig. 17 shows the captured snapshots of the network and when the $\delta \leq 1$ the noise current doesn't have any effect of the network and hence we could note the obstacle induced wave re-entry and spiral waves as in Fig.17a. But as we increase the noise variance, the obstacle effects are soon supressed by the noise current and we could note the breaking of the spiral waves. The addition of noise induces random excitability in the network and hence the media enters into state of high excitability thus supporting simple wave propagation. The number of spiral seeds reduce as we increase the noise variance and finally the network goes unstable for $\delta>10$.
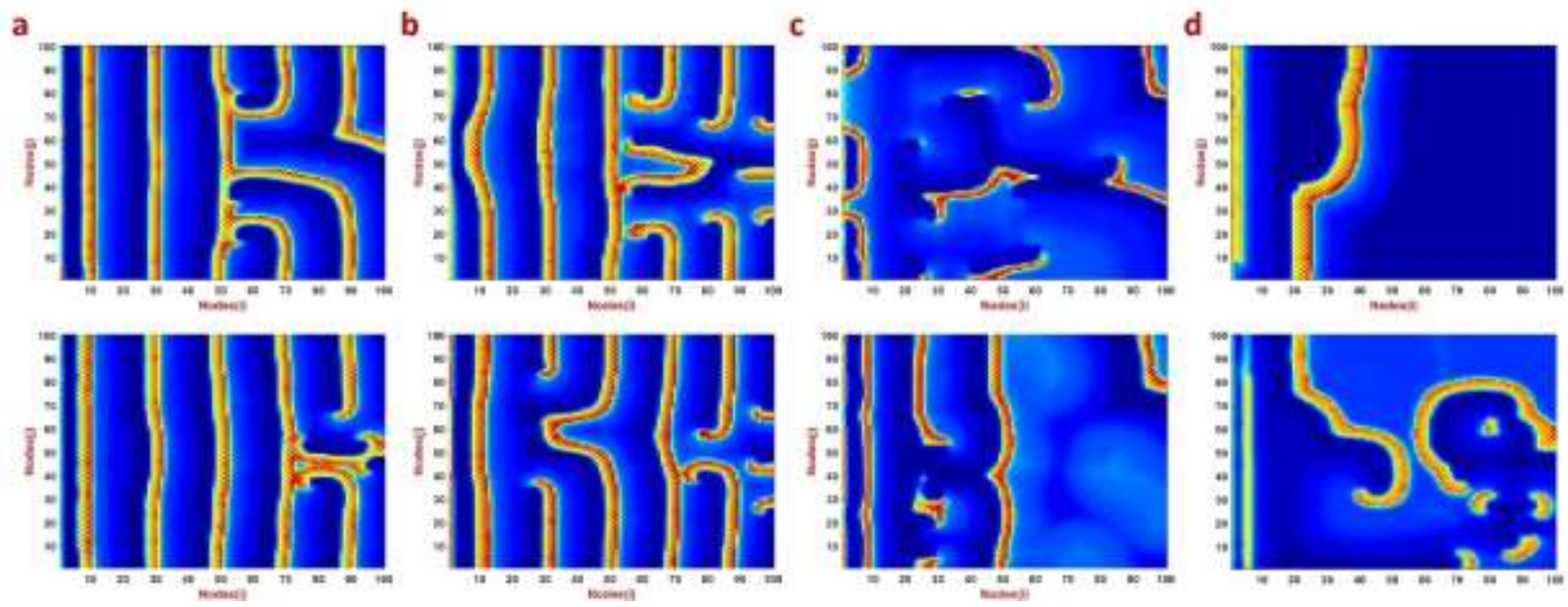

Fig.17: Spatiotemporal behaviour of the two-layer DHBN network considering noise presence in both the layers while there are two different size and orientation of the obstacles considered. The 
stimulus is applied to both the layers and the sub figures [a,b,c,d] shows the snapshots of the two layers for various values of the noise variance $[1,5,8,10]$ respectively.

\section{Results and Discussions:}

A discrete neuron map derived by modifying the continuous time Huber-Braun neuron model is proposed in this paper. The novelty of the model is the inclusion of two slow, subthreshold currents along with the leak and channel currents. We have considered the temperature effect on various current channels of the neurons and depending on the temperature the neuron shows periodic spiking and chaotic bursting behaviour. The bifurcation studies reveal the different periodic and chaotic regime of the DHBN model. To clearly show the chaotic and periodic regime in a $2 \mathrm{D}$ parametric space, we have used the Lyapunov exponents-based chaos diagram. A sample entropy algorithm is proposed to measure the complexity of the DHBN model which is correlated with the Lyapunov exponent chaos diagram.

Though nodal dynamics will make us understand the complete behavioural pattern of the neuron, it is the collective dynamics which plays a significant role in biophysical models. Hence we constructed a simple 2D network of DHBN modes and investigated the network behaviour by applying a periodic force. The network shows simple plane wave propagation with no sign of wave re-entry on spiral waves. Now our interest is to investigate the conditions which can induce wave re-entry. Referring literature helped us to identify two major re-entry simulation situations. The first is by introducing an obstacle in the network and the second is introducing noise. We first decided to investigate the obstacle effects and for this we have chosen two orientations and six different sizes of obstacle. The observation shows that when the obstacle is parallel to the wave entry, the effects and significant way and to the obstacle a plane perpendicular to the wave entry. Also, we have shown the minimum dimensions of the obstacles which can cause wave re-entry and induce spiral waves. To check the obstacle effects on multilayer networks, we constructed a two-layer DHBN network. Firstly, we showed that though the obstacle is considered only in one layer, the stimulus applied to the layers play an important role is the wave re-entry in the layers. This is proved by setting only stimulus and obstacles to the first layer, but the second layer also displays stimulus wave re-entry like the first layer. This coherent behaviour is removed when we apply stimulus to the second layer also.

In the next discussion we investigate the influence of noise on inducing spiral waves in the network under no noise and no obstacle condition the 2D DHBN network shows no wave re-entry but when exposed to noise whose variance is $\delta>1$, the network shows multiple spiral seeds. Thus, we could confirm that noise exposure changes the excitabilities of the neurons. Now we consider noise in the presence of a parallel obstacle in the network. Now as the noise variance is increased, the spiral waves break into multiple spiral seeds and for larger noise variance the nodes lose resetting at this remains in the high amplitude state (idle) without spiking or bursting phenomenon. Similar effects of noise can be observed in the two-layer DHBN network. Additionally, we could show that noise effects on a parallel obstacle are more than the perpendicular obstacles.

Conflict of Interest: The authors declare that they have no conflict of interest. 
Funding: Acknowledgement: This work is partially funded by Centre for Nonlinear Systems, Chennai Institute of Technology, India vide funding number CIT/CNS/2020/RD/064

\section{References:}

[1] A. L. Shilnikov, Rulkov, N. F.: Subthreshold oscillations in a mapbased neuron model., Phy.Lett.A, vol. 328, pp. 177-184, (2004).

[2] N. F. Rulkov.: Modeling of spiking-bursting neural behavior using twodimensional map, Phy.Rev.E, vol. 65, p. 041922, (2002).

[3] J. C. A. Pontes, Viana, R. L., Lopes, S. R., Batista, C. A. S., Batista, A. M.: Bursting synchronization in non-locally coupled maps., Physica A, vol. 387, pp. 4417-4428, (2008).

[4] O. Kinouchi, Tragtenberg, M. H. R.: Modeling neurons by simple maps., Int. J. Bifurcat. Chaos, vol. 6, pp. 2343-2360, (1996).

[5] E. M. Izhikevich, Hoppensteadt, F.: Classification of bursting mappings., Int. J. Bifurcat. Chaos, vol. 14, pp. 3847-3854, (2004).

[6] K. Kaneko.: Theory and Applications of Coupled Map Lattices. New York, NY: Wiley, 1993.

[7] G. Tanaka, Ibarz, B., Sanjuán, M. A. F., Aihara, K.: Synchronization and propagation of bursts in networks of coupled map neurons., Chaos, vol. 16, p. 013113, (2006).

[8] J. M. C. B. Ibarz, M.A.F. Sanjuán.: Map-based models in neuronal dynamics, Physics Reports, vol. 501, pp. 1-74, (2011).

[9] G. De Vries.: Collective dynamics in sparse networks, Phys. Rev. Lett., vol. 109, p. 138103, (2012).

[10] P. A. A. A. Kotini.: Dynamics of noisy neural nets with chemical markers and gaussiandistributed connectivities, Conn. Sci., vol. 9, pp. 381-404, (1997).

[11] X. S. L. D.Q. Wei.: Ordering spatiotemporal chaos in discrete neural networks with smallworld connections, Europhys. Lett., vol. 77, p. 68004, (2007).

[12] M. T. H. H.A.Braun, N.Anthes, K.Voigt, A.Neiman, X.Pei, F.Moss.: Noise-induced impulse pattern modifications at different dynamical period-one situations in a computer model of temperature encoding, Bio Systems, vol. 62, pp. 99-112, (2001).

[13] C. F. S.Postnova, W.Y.Jin,H.Schneider,H.Braun.: A computational study of the interdependencies between neuronal impulse pattern, noise effects and synchronization, J.Physiol., vol. 104, pp. 176-189, (2010).

[14] S. P. C. Finkea, E. Rosa Jr, J.A. Freund, M.T. Huber, K. Voigt, F.E. Moss, H.A. Braun, U. Feudel.: Noisy activation kinetics induces bursting in the Huber-Braun neuron model, Eur. Phys. J. Special Topics, vol. 187, pp. 199-203, (2010).

[15] Z. D. Q.Y. Wang, M. Perc, G. Chen.: Synchronization transitions on small-world neuronal networks: effects of information transmission delay and rewiring probability, Europhys. Lett., vol. 83, p. 50008, (2008).

[16] H. Zou, Guan, S., Lai, C.-H.: Dynamical formation of stable irregular transients in discontinuous map systems., Phys Rev E, vol. 80, p. 046214, (2009).

[17] B. R. R. B. R. C. Budzinski, T. L. Prado, S. R. Lopes.: Temperature dependence of phase and spike synchronization of neural networks, Chaos, Solitons \& Fractals, vol. 123, pp. 3542, (2019). 
[18] C. F. Finke, Jan \& Rosa, Epaminondas \& Braun, Hans \& Feudel, Ulrike.: On the role of subthreshold currents in the Huber-Braun cold receptor model, Chaos, vol. 20, (2010).

[19] H. H. Braun, M \& Anthes, N \& Voigt, K \& Neiman, Alexander \& Pei, X \& Moss, F.: Noise-induced impulse pattern modifications at different dynamical period-one situations in a computer model of temperature encoding., Bio Systems, vol. 62, pp. 99-112, (2001).

[20] J. S. Wang, Liu \& H., Wang.:Dynamical Properties of Firing Patterns in the Huber-Braun Cold Receptor Model in Response to External Current Stimuli. ," Neural Network World vol. 25, pp. 641-655, (2015).

[21] J. A. F. Christian Finke, Epaminondas Rosa Jr, Paul H. Bryant, Hans A. Braun, Ulrike Feudel.: Temperature-dependent stochastic dynamics of the Huber-Braun neuron model, Chaos, vol. 21, p. 047510, (2011).

[22] K. J. McGahan K.: A mathematical model analyzing temperature threhold dependence in cold sensitive neurons., PLoS One, vol. 15, p. e0237347, (2020).

[23] H. W. H. A. Braun, K. Scha“fer, and M. C. Hirsch,: Oscillation and noise determine signal transduction in shark multimodal sensory cells, Nature, vol. 367, pp. 270-273, (1994).

[24] M. V. Ivanchenko, Osipov, G. V., Shalfeev, V. D., Kurths.: Network mechanism for burst generation, Phys. Rev. Lett., vol. 98, p. 108101, (2007).

[25] M. Bazhenov, Rulkov, N. F., Fellous, J., Timofeev, I.: Role of network dynamics in shaping spike timing reliability, Phy.Rev.E, vol. 72, p. 041903, (2005).

[26] D. H. Yao Y, Yi M, Ma J., Impact of bounded noise on the formation and instability of spiral wave in a 2D Lattice of neurons, Sci Rep, vol. 7, p. 43151, (2017).

[27] J. V. Christian Finke, Svetlana Postnova, Hans Albert Braun.: Propagation effects of current and conductance noise in a model neuron with subthreshold oscillations, Mathematical Biosciences, vol. 214, pp. 109-121, (2008).

[28] Q. Y. Wang, Duan, Z., Perc, M., Chen, G. R.: Synchonization transitions on small-world neuronal networks: effects of information transmission delay and rewiring probability., Europhys. Lett., vol. 83, p. 50008, (2008).

[29] E.M.Izhikevich.: Neural excitability, spiking and bursting, Int. J . Bifurc. Chaos, vol. 10, pp. 1171-1266, (2000).

[30] L. Fei Tan, Junwei Lu, YumingChu.: Analysis of fixed-time outer synchronization for double-layered neuron-based networks with uncertain parameters and delays, Journal of the Franklin Institute, vol. 357, 2020.

[31] R. Kamimura.: Neural self-compressor: Collective interpretation by compressing multilayered neural networks into non-layered networks, Neurocomputing, vol. 323, pp. 12-36, (2019).

[32] Z. C. Tianshun Wang, RuiBu, RunshengMa.: Stability and Hopf bifurcation analysis of a simplified six-neuron tridiagonal two-layer neural network model with delays, Elsevier Neurocomputing, vol. 332, pp. 203-214, (2019).

[33] R. L. V. J.C.A. de Pontes, S.R. Lopes, C.A.S. Batista, A.M. Batista.: Bursting synchronization in non-locally coupled maps, Physica A, vol. 387, pp. 4417-4428, (2008).

[34] G. T. B. Ibarz, M.A.F. Sanjuán, K. Aihara.: Sensitivity versus resonance in simple mapbased conductance neuron models, Phys. Rev. E, vol. 75, p. 041902, (2007).

[35] B. W. Yong Wu, Xiaoxiao Zhang and Hao Chen.: Spiral wave of a two-layer coupling neuronal network with multi-area channels, International Journal of Modern Physics B, vol. 33, p. 1950354, (2019). 
[36] A. J. M. K. Yu Feng, Fawaz E. Alsaadi, Tasawar Hayat, Viet-Thanh Pham.: Spiral wave in a two-layer neuronal network, Eur.Phys.H.Special Topics, vol. 228, pp. 2371-2379, (2019).

[37] S. R. L. T. L. Prado, C. A. S. Batista, J. Kurths, R. L. Viana.: Synchronization of bursting hodgkin-huxley-type neurons in clustered networks, Physical Review E, vol. 90, p. 032818, (2014).

[38] A. J. M. K. Karthikeyan Rajagopal, Fatemeh Parastesh, Irene Moroz, Anitha Karthikeyan, Sajad Jafari, Dynamical behavior and network analysis of an extended Hindmarsh-Rose neuron model, Nonlinear Dyn vol. 98, p. 477, (2019).

[39] F. P. Karthikeyan Rajagopal, Hamed Azarnoush, Boshra Hatef, Sajad Jafari and Vesna Berec.: Spiral waves in externally excited neuronal network: solvable model with a monotonically differentiable magnetic flux, Chaos: An Interdisciplinary Journal of Nonlinear Science, vol. 29, (2019).

[40] H. Z. G. Yuan, X. Wang, G. Wang, S. Chen.: Feedback-controlled dynamics of spiral waves in the complex Ginzburg-Landau equation, Nonlinear Dyn, vol. 90, pp. 27452753, (2017).

[41] V.-T. P. Z. Rostami , S. Jafari , F. Hadaeghi , J. Ma.: Taking control of initiated propagating wave in a neuronal network using magnetic radiation, Appl. Math. Comput., vol. 338, pp. 141-151, (2018).

[42] D. S. Y. Wang, X. Gao , S.-X. Qu , Y.-C. Lai , X. Wang.: Effect of network structural perturbations on spiral wave patterns, Nonlinear Dyn, vol. 93, pp. 1-10, (2018).

[43] I. F. R. Majumder, A.S. Teplenin , A .A . de Vries , A .V. Panfilov , D.A . Pijnappels.: Optogenetics enables real-time spatiotemporal control over spiral wave dynamics in an excitable cardiac system, eLife, vol. 7, p. e41076, (2018).

[44] K. R. Zahra Rostami, Abdul Jalil M. Khalaf, Sajad Jafari, Matjaž Perc, Mitja Slavinec.: Wavefront-obstacle interactions and the initiation of reentry in excitable media, Physica A: Statistical Mechanics and its Applications, vol. 509, pp. 1162-1173, (2018).

[45] Y. Z. Josef M. Starobin, Elizabeth M. Rusnak, C. Frank Starmer.: Wavelet Formation in Excitable Cardiac Tissue: The Role of Wavefront-Obstacle Interactions in Initiating HighFrequency Fibrillatory-Like Arrhythmias, Biophysical Journal, vol. 70, pp. 581-594, (1996).

[46] W. X. Xiaoying Huang, Jianmin Liang, Kentaroh Takagaki, Xin Gao, Jian-young Wu.: Spiral Wave Dynamics in Neocortex, Neuron, vol. 68, pp. 978-990, 2010.

[47] H. A. Braun, Huber, M. T., Anthes, N., Voigt, K., Neiman, A., Pei, X. \& Moss, F.: Interactions between slow and fast conductances in the Huber/Braun model of cold receptor discharges, Neurocomputing, pp. 32-33, (2000).

[48] L. W. J. K. Douglass, E. Pantazelou, and F. Moss.: Noise enhancement of information transfer in crayfish mechanoreceptors by stochastic resonance, Nature vol. 365, pp. 337340, (1993).

[49] J. A. F. Christian Finke, Epaminondas Rosa Jr, Paul H. Bryant, Hans A. Braun, and Ulrike Feudel.: Temperature-dependent stochastic dynamics of the Huber-Braun neuron model, Chaos, vol. 21, p. 047510, (2011). 


\section{Figures}
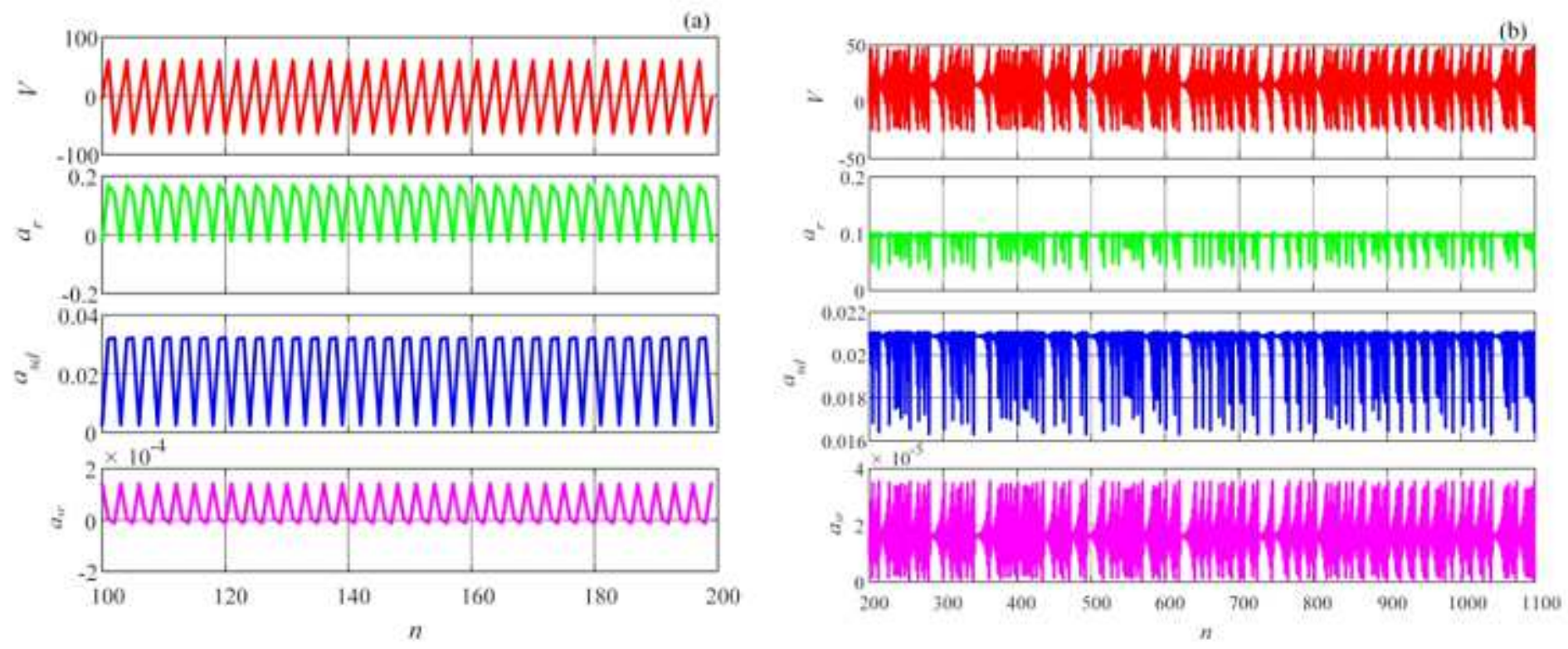

Figure 1

Time series of the system with different parameters. (a) $\mathrm{T}=15$; (b) $\mathrm{T}=11$.
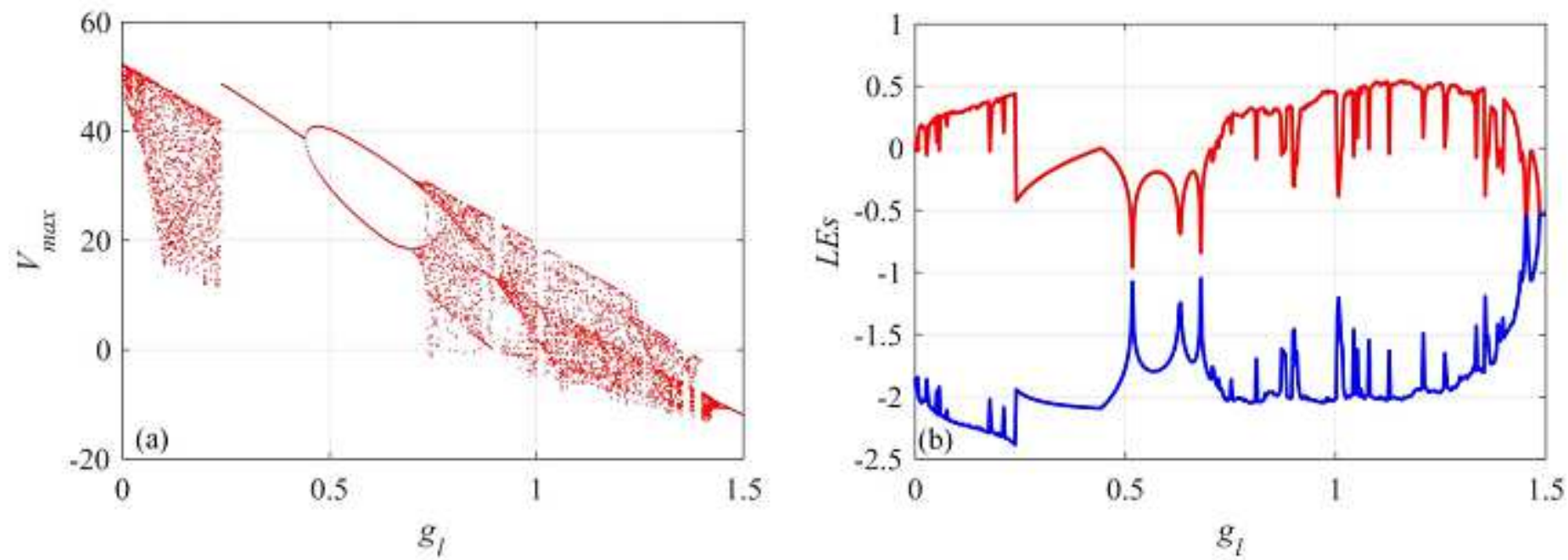

Figure 2

Dynamics of the system with the variation of parameter g_l. (a) Bifurcation diagram; (b) Lyapunov exponents 

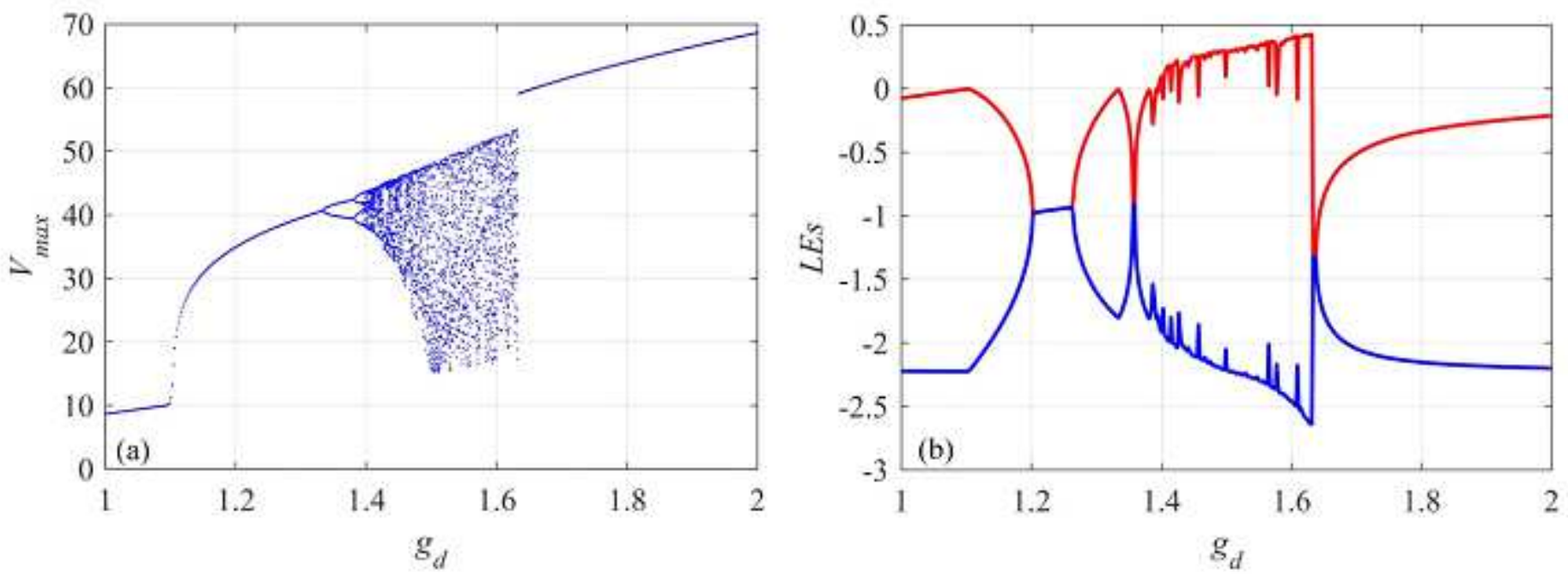

Figure 3

Dynamics of the system with the variation of parameter gd. (a) Bifurcation diagram; (b) Lyapunov exponents.
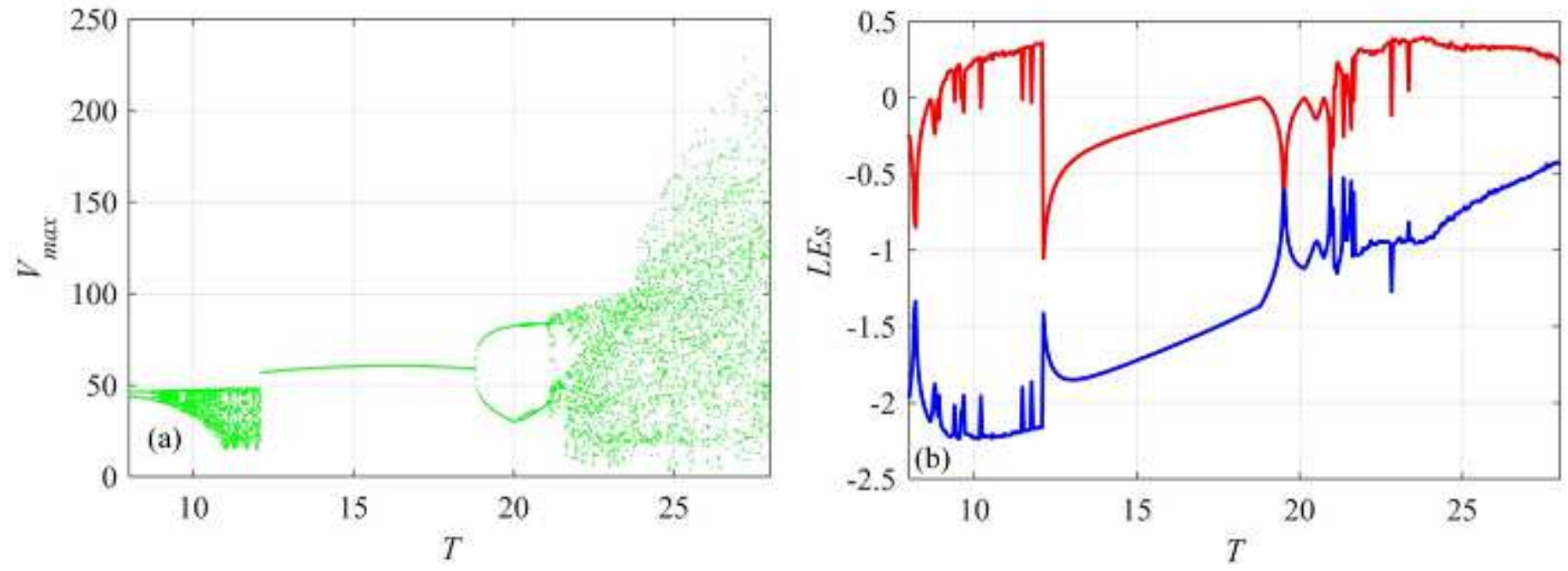

Figure 4

Dynamics of the system with the variation of parameter T. (a) Bifurcation diagram; (b) Lyapunov exponents. 
(a)

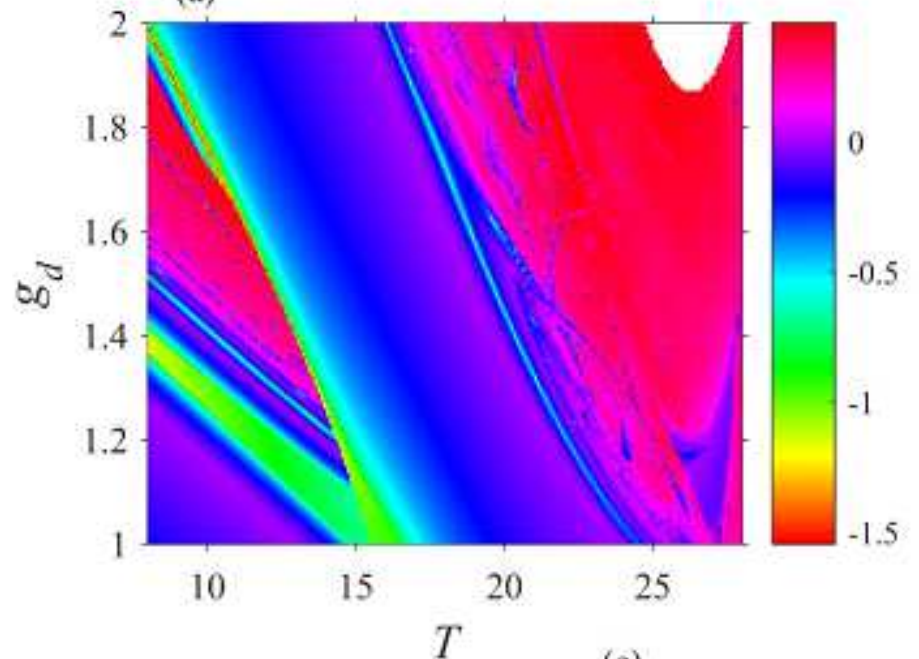

(c)

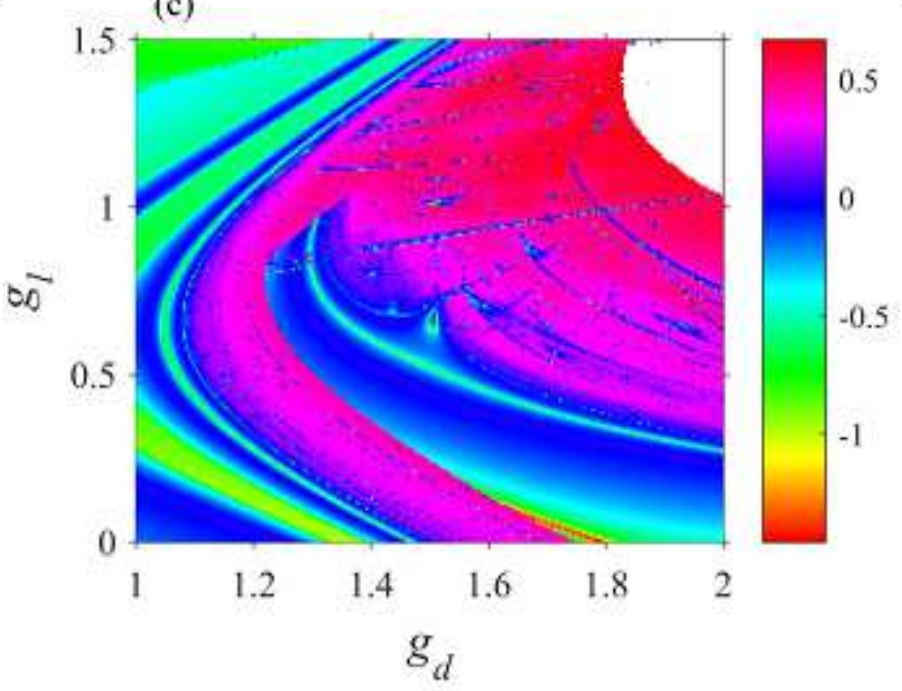

(b)

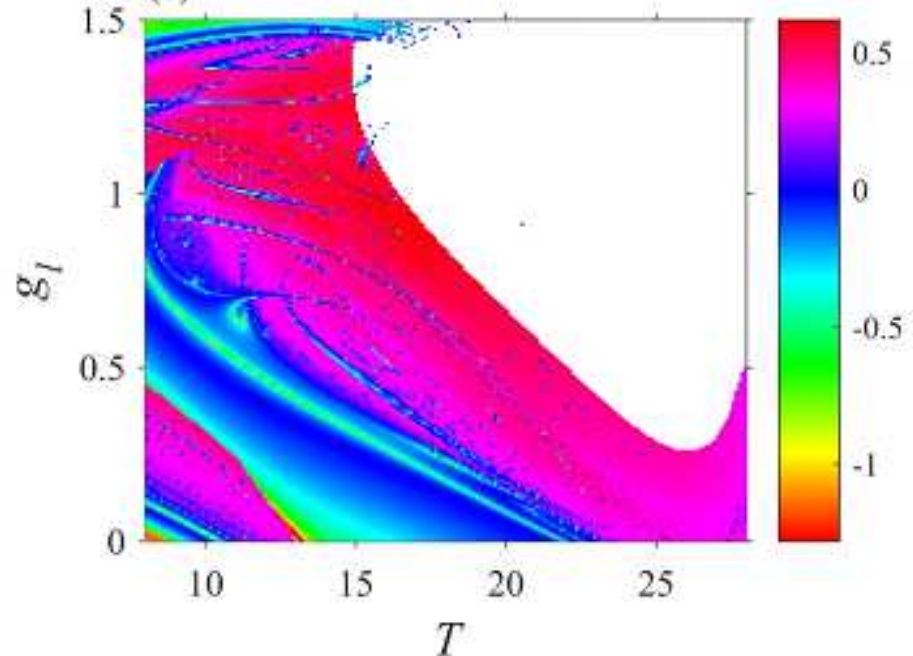

Figure 5

See the Manuscript Files section for the complete figure caption. 

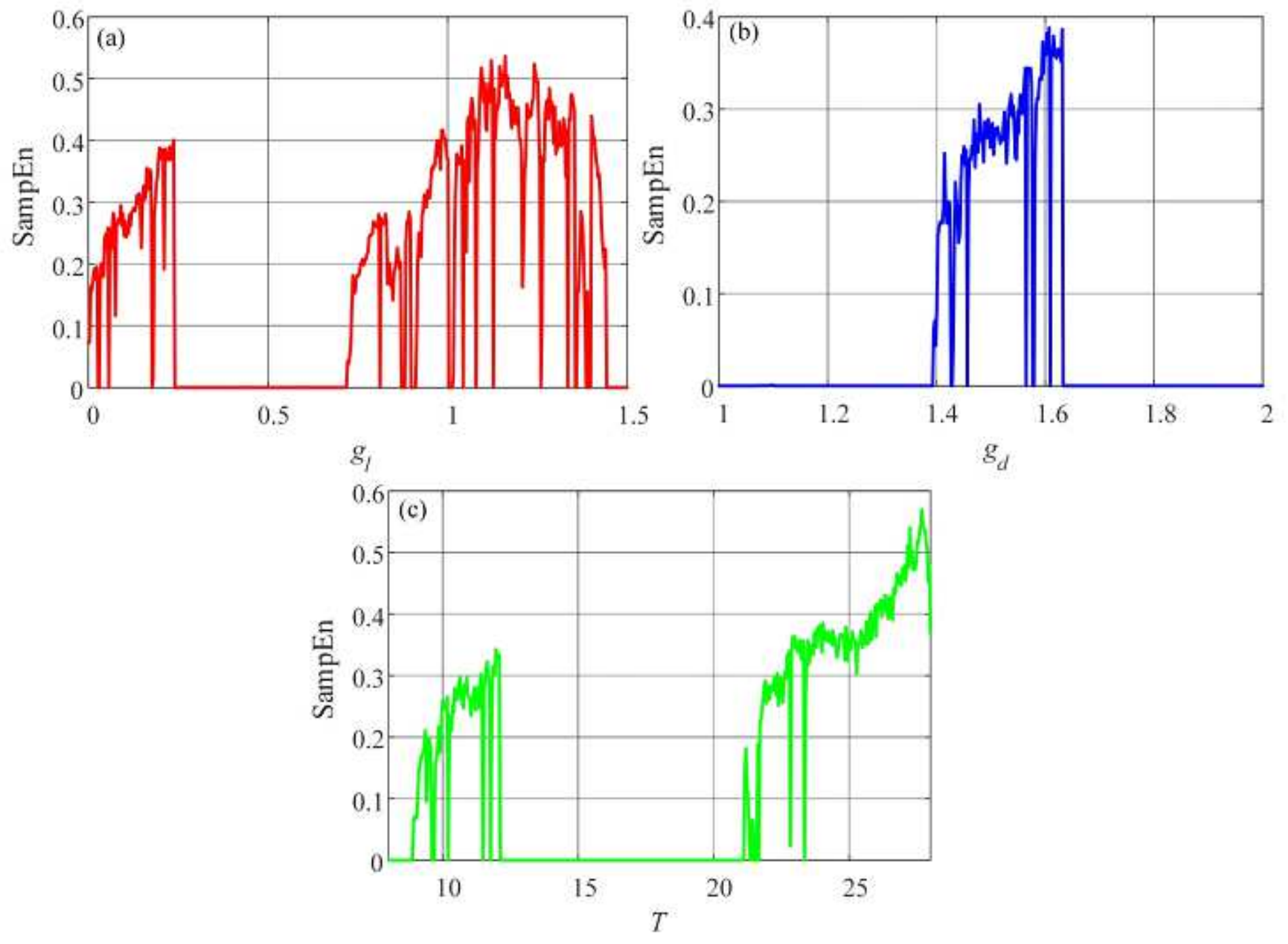

Figure 6

SampEn complexity analysis results with different parameters. (a) gl varying; (b) gd varying; (c) T varying 


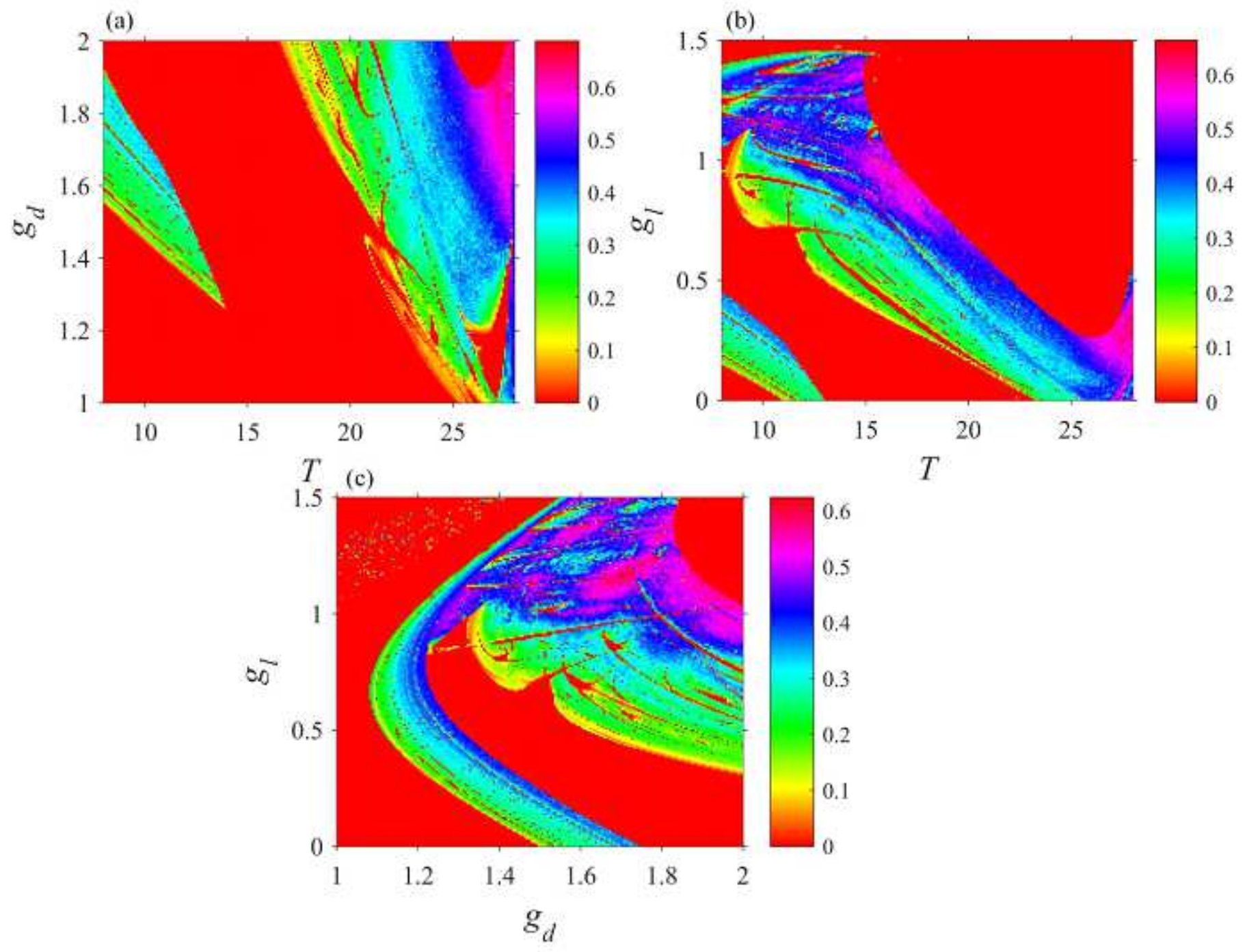

Figure 7

SampEn based chaos diagrams in different parameter planes. (a) T-gd; (b) T-gl; (c) gd-gl 


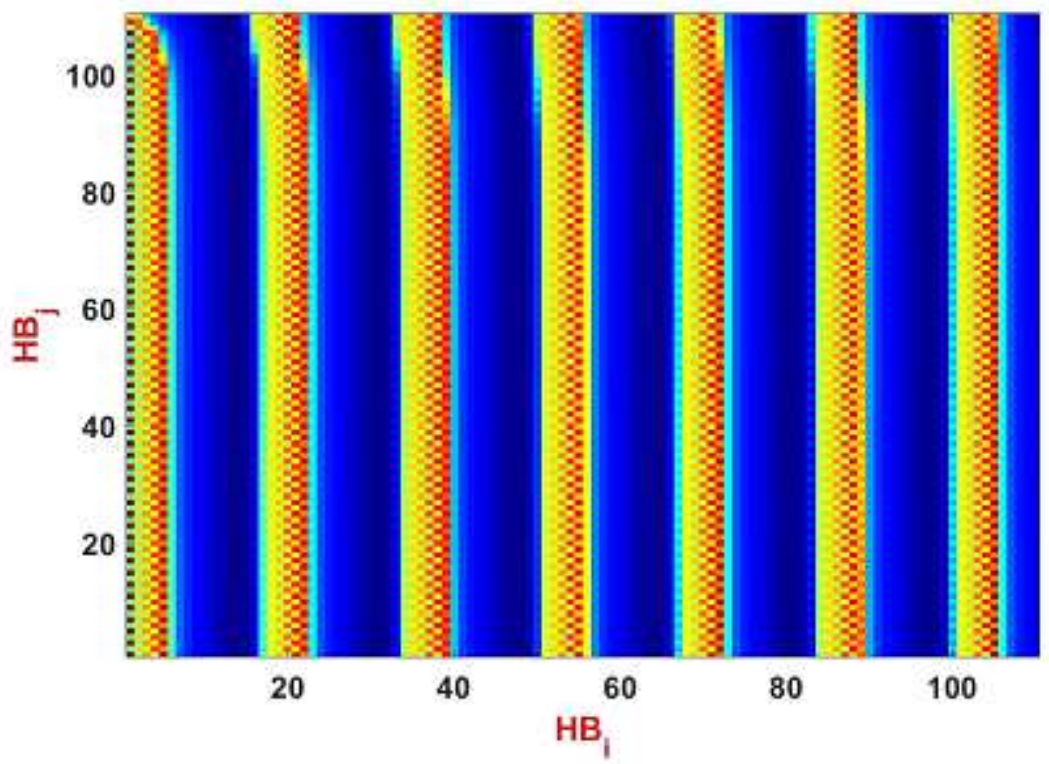

\section{Figure 8}

Travelling waves seen in the DHBN network excited by a periodic stimuli. The amplitude and frequency of the stimuli are 0.1 and 0.01 respectively. The coupling strength $\sigma=1$ and we considered no flux boundary conditions for the network.
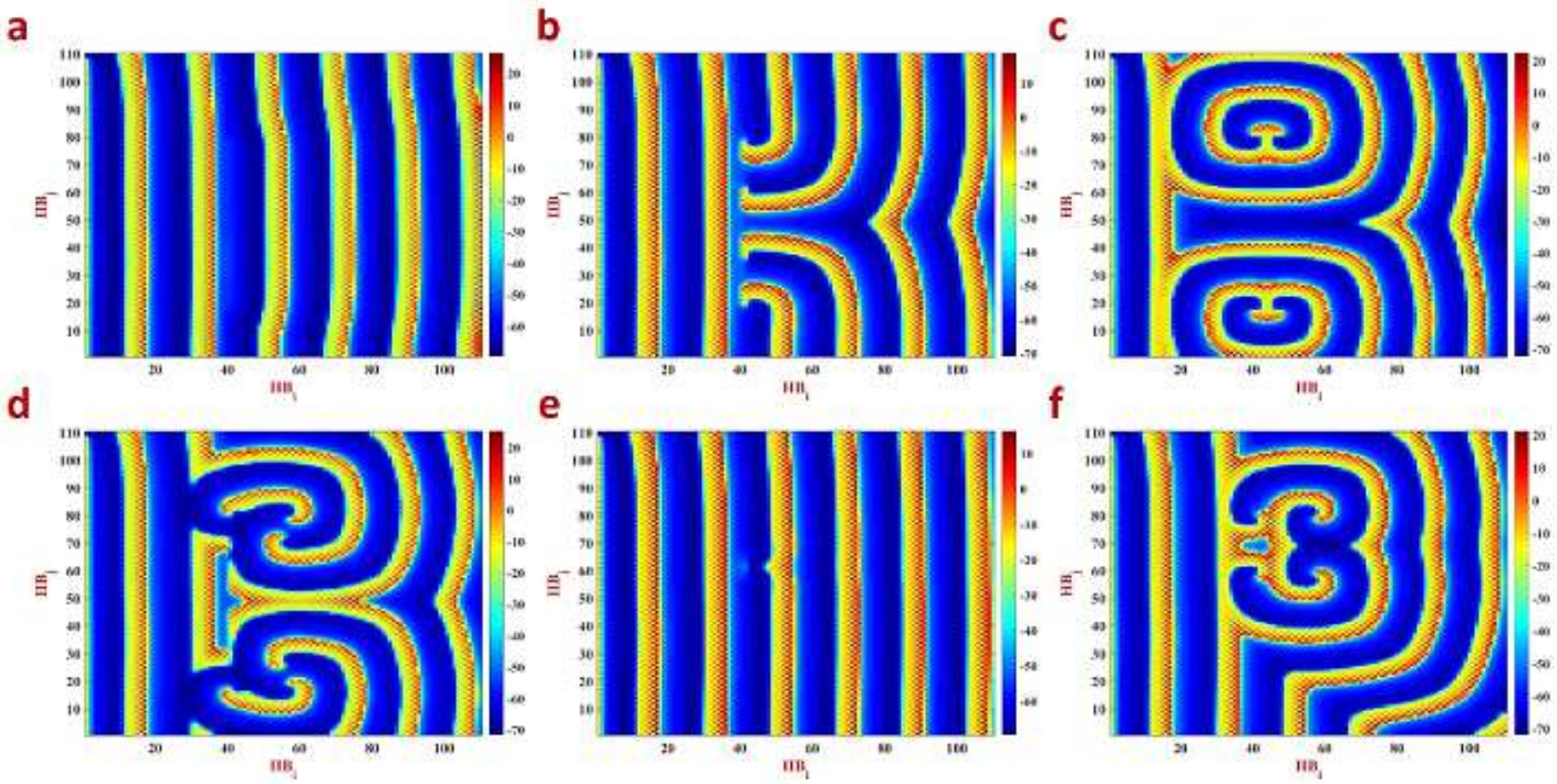

\section{Figure 9}

Spatiotemporal behaviour of the DHBN network considering the obstacle parallel to the wave front. Different sized obstacles are used for the analysis with obstacles greater than five node width we could 
note that wave re-entry and spiral waves are formed near the boundary opposite to the wave entry side.

a

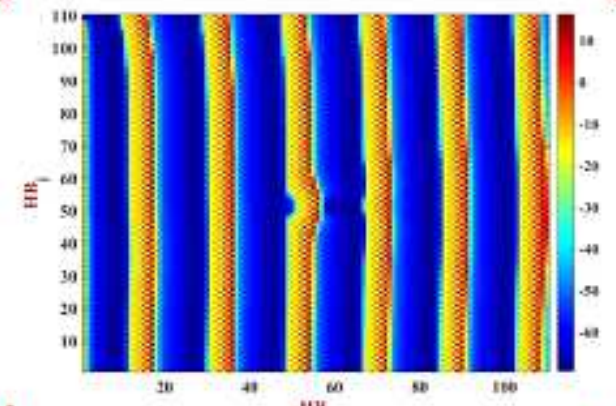

d

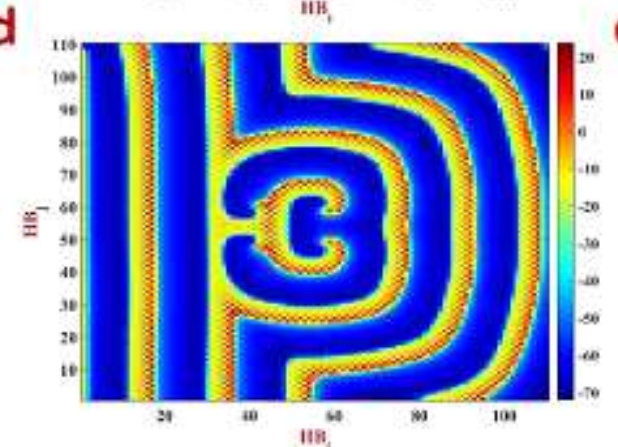

b
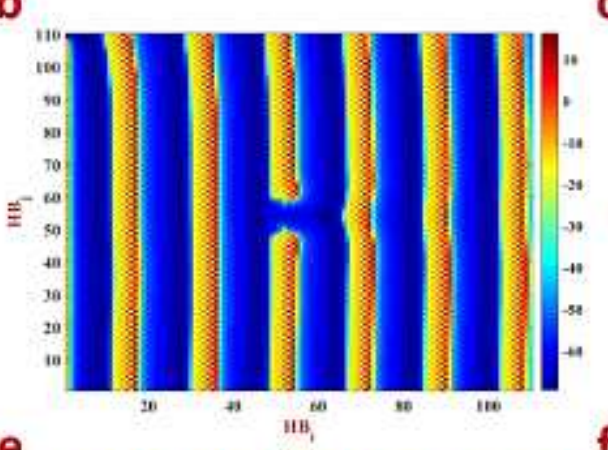
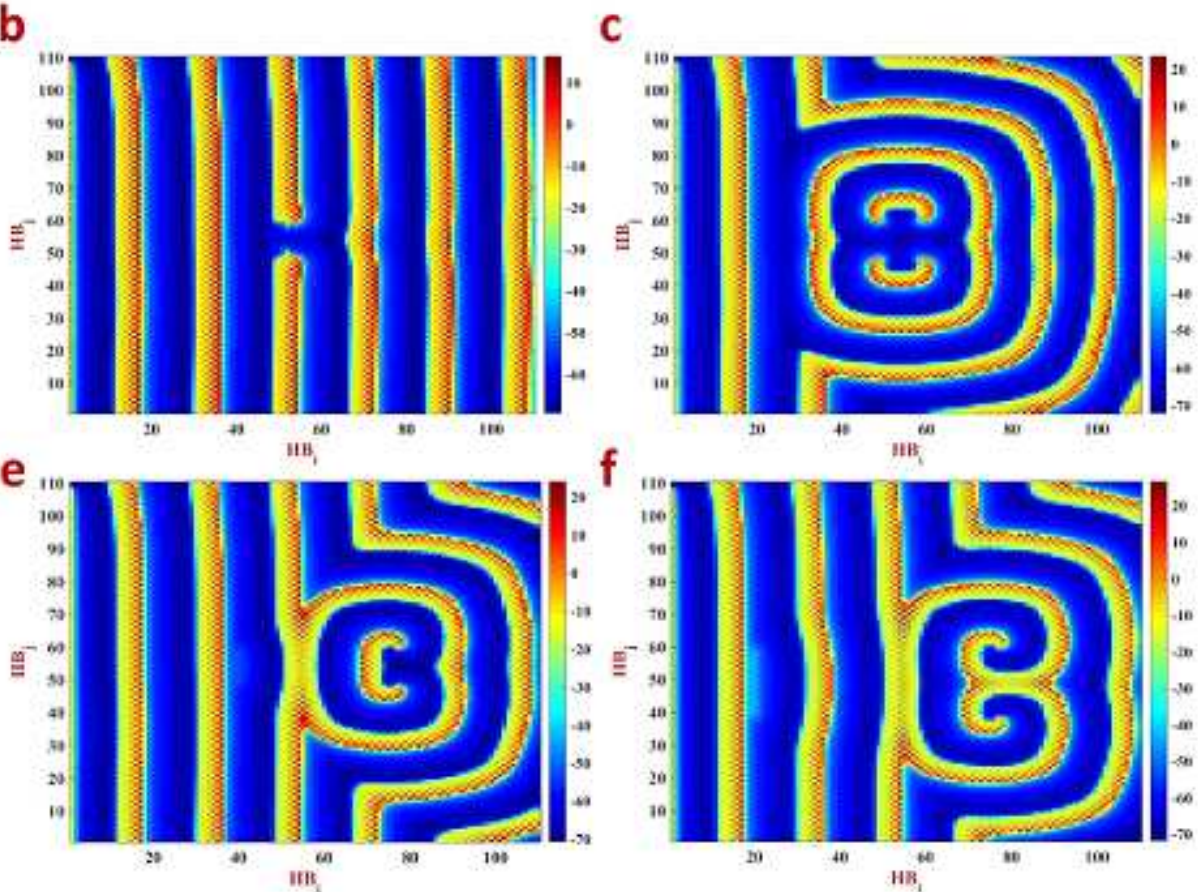

Figure 10

Snapshots of the media with obstacle perpendicular to the wave entry. Different combinations of width and length of the obstacle are considered with system parameters and stimuli settings same as used in Fig.8.
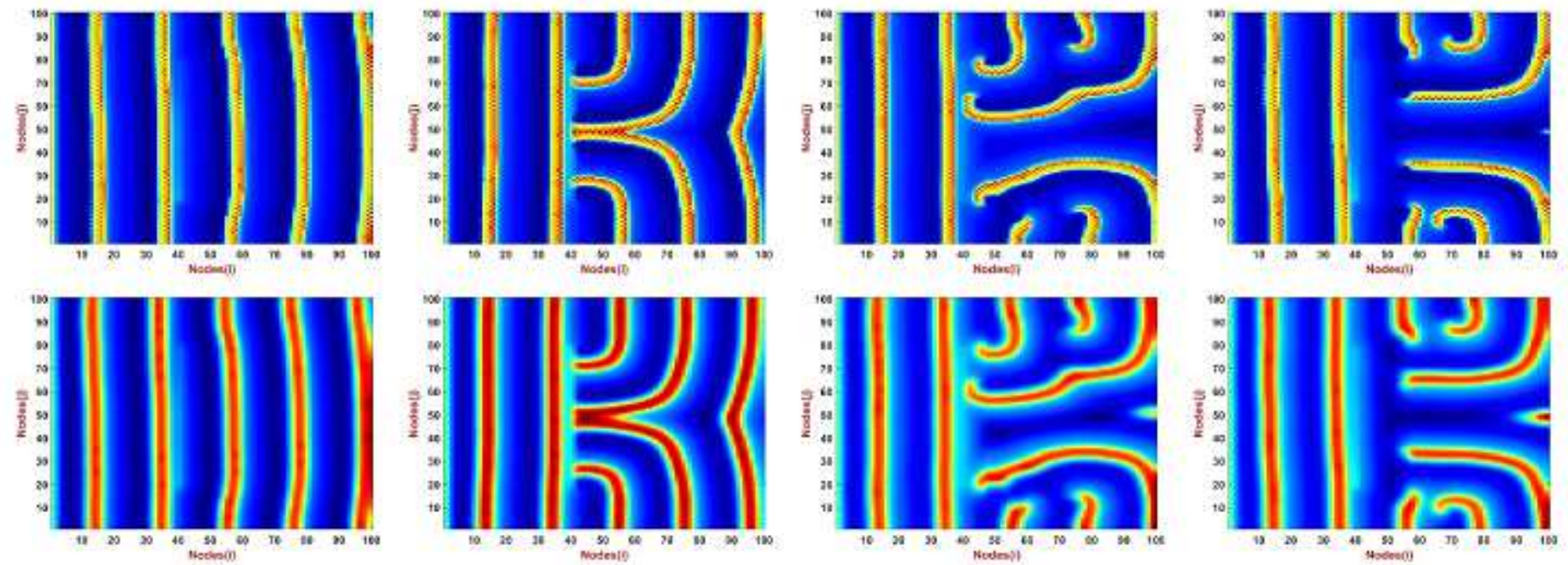

\section{Figure 11}

Spatiotemporal snapshots of the two-layer DHBN network with the obstacle and stimulus applied to the upper layer while the lower layer is coupled to the upper layer with a coupling strength of 0.05 . 

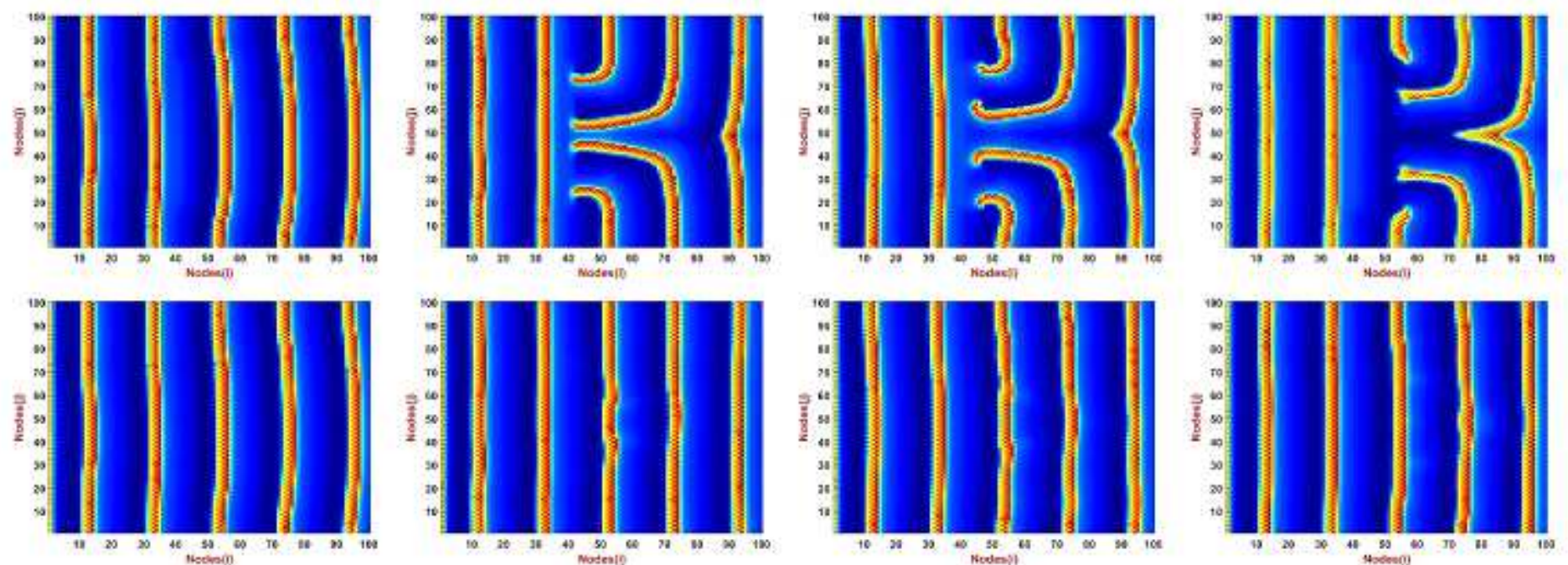

Figure 12

The snapshots of the two-layer network with stimulus applied to both the layers while the obstacle is considered only in the upper layer. The stimulus and coupling strength settings are similar to Fig.11.
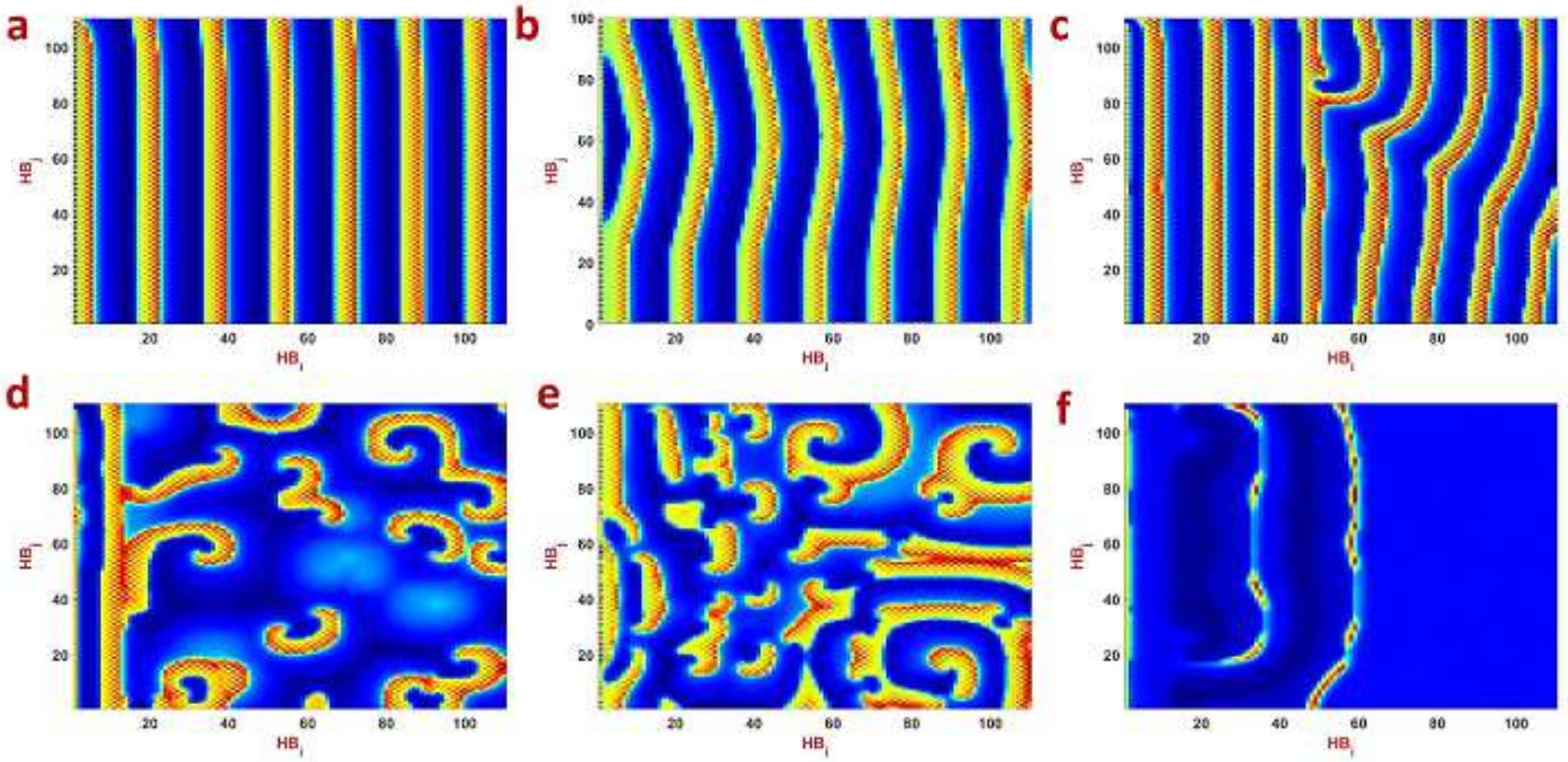

\section{Figure 13}

Spatiotemporal snapshots of the single layer network without obstacles for different noise variances $[0,1,3,5,7,10]$ displayed with figure labels $[a, b, c, d, e, f]$ respectively. 


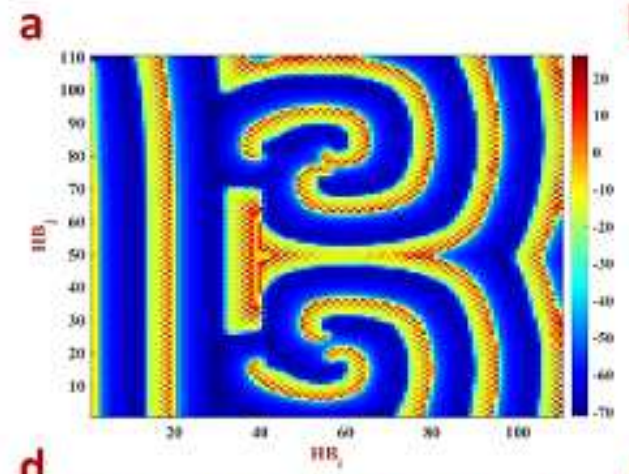

d

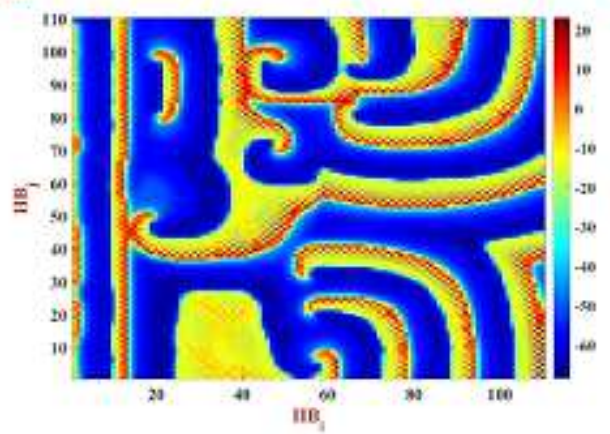

b

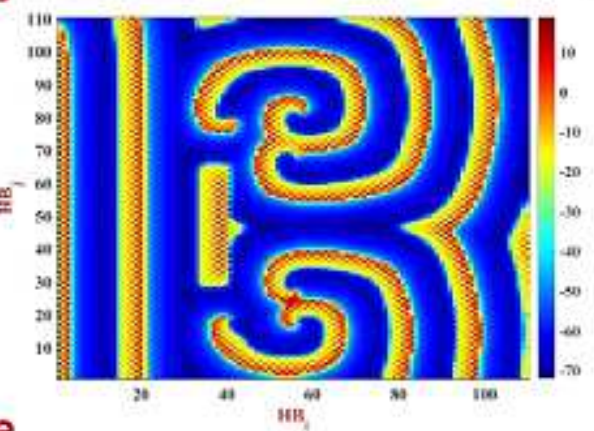

e

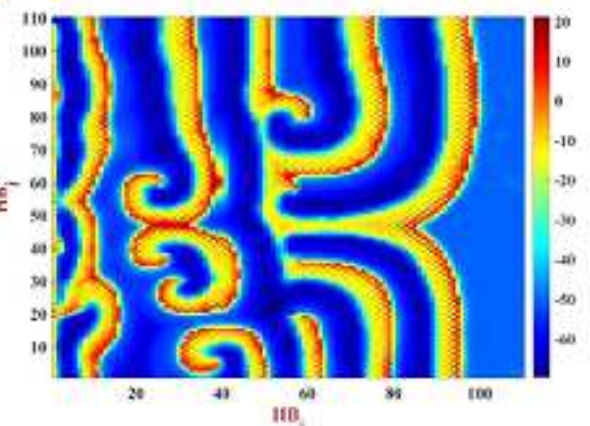

c

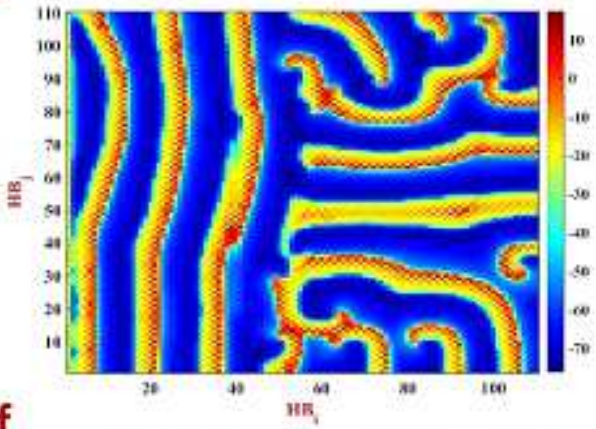

f

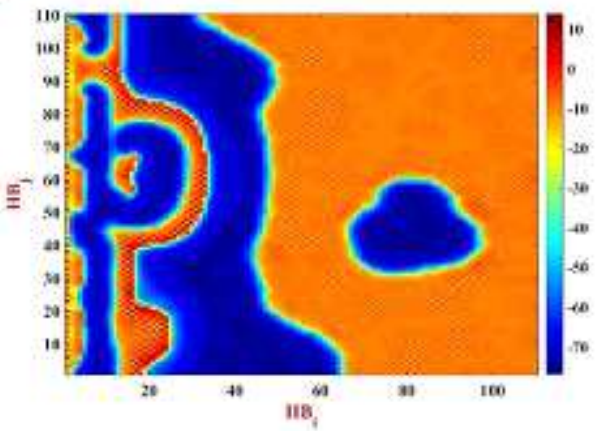

Figure 14

Spatiotemporal snapshots of the single layer network with obstacle as used in Fig.9d for different noise variances $[0.5,1,5,7,10,12]$ displayed with figure labels $[a, b, c, d, e, f]$ respectively. The obstacle is placed parallel to the wave entry.
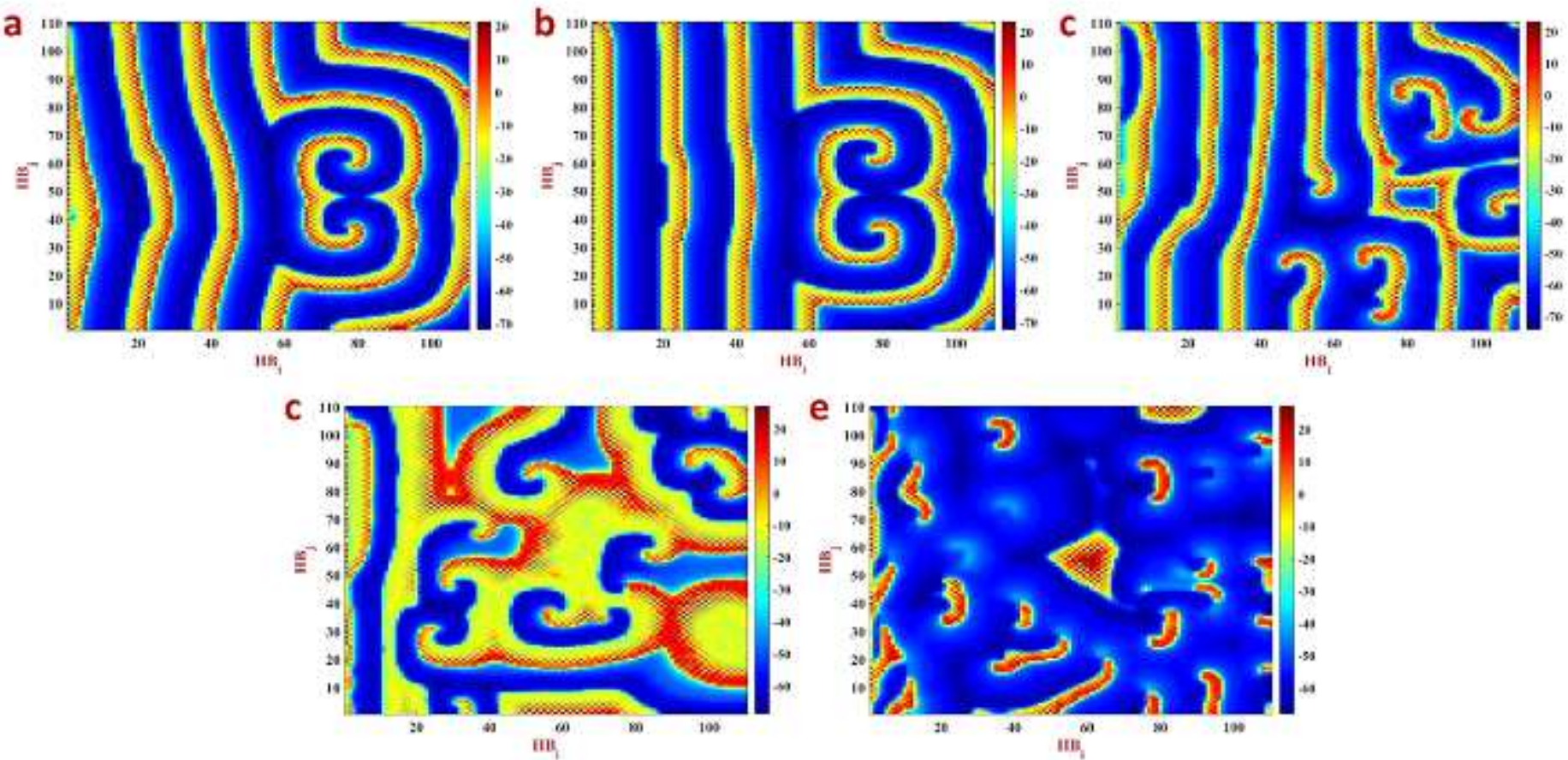

Figure 15 
Spatiotemporal snapshots of the single layer network with obstacle as used in Fig.9d for different noise variances $[0.5,1,5,7,9]$ displayed with figure labels $[a, b, c, d, e]$ respectively. The obstacle is placed perpendicular to the wave entry.
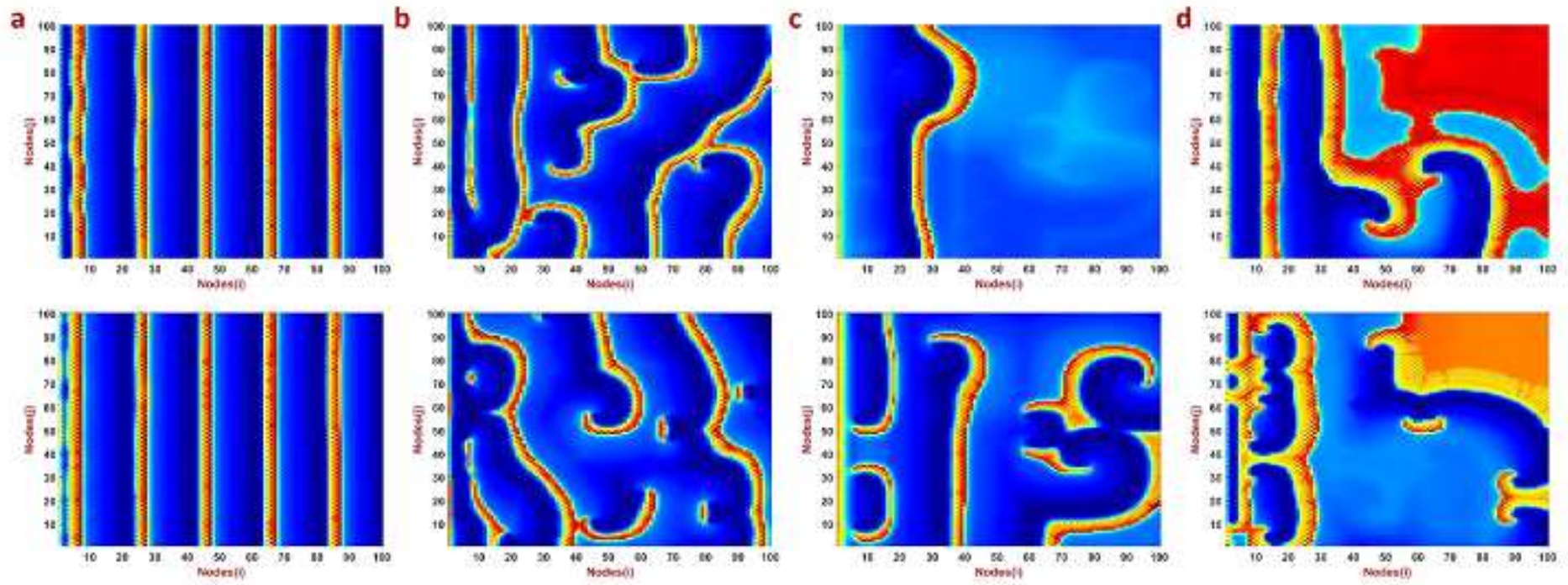

Figure 16

Spatiotemporal behaviour of the two-layer DHBN network considering noise presence in both the layers while there is no obstacle considered. The stimulus is applied to both the layers and the sub figures $[a, b, c, d]$ shows the snapshots of the two layers for various values of the noise variance $[1,5,8,10]$ respectively.

a
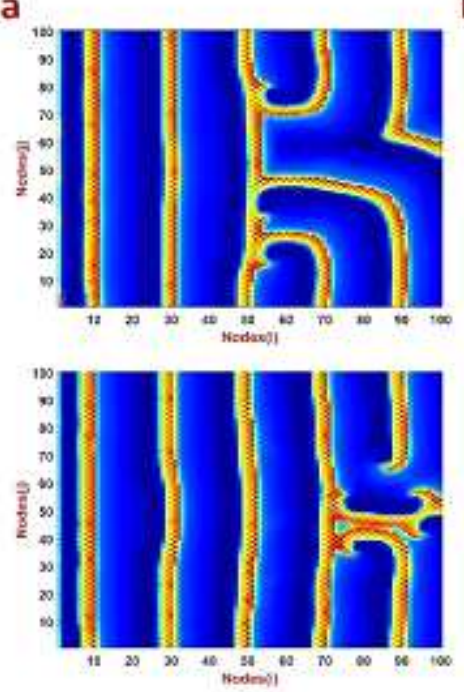

b
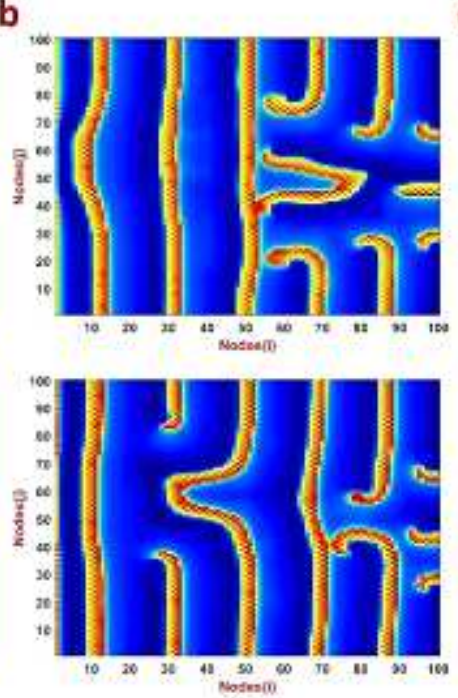

c
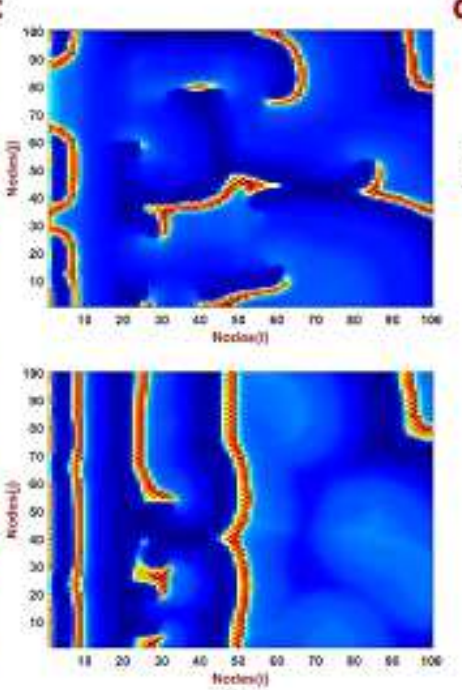

d
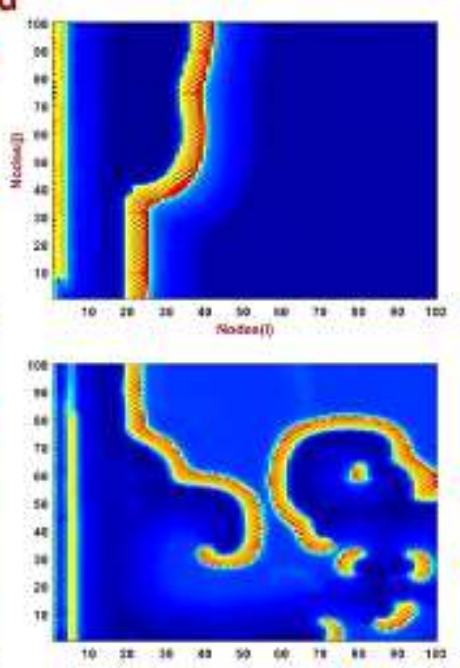

Figure 17

Spatiotemporal behaviour of the two-layer DHBN network considering noise presence in both the layers while there are two different size and orientation of the obstacles considered. The stimulus is applied to 
both the layers and the sub figures $[a, b, c, d]$ shows the snapshots of the two layers for various values of the noise variance $[1,5,8,10]$ respectively. 